ARTICLE

\title{
The intellectual disability protein RAB39B selectively regulates GluA2 trafficking to determine synaptic AMPAR composition
}

\author{
Maria Lidia Mignogna ${ }^{1,2,3, \star}$, Maila Giannandrea ${ }^{1,2, \star}$, Antonia Gurgone ${ }^{1,3}$, Francesca Fanelli ${ }^{4}$, Francesco Raimondi ${ }^{4}$, \\ Lisa Mapelli ${ }^{5}$, Silvia Bassani ${ }^{5}$, Huaqiang Fang ${ }^{6}$, Eelco Van Anken7, Massimo Alessio ${ }^{7}$, Maria Passafaro ${ }^{5}$, \\ Silvia Gatti ${ }^{2}$, José A. Esteban ${ }^{8}$, Richard Huganir ${ }^{6}$ \& Patrizia D'Adamo ${ }^{1}$
}

RAB39B is a member of the RAB family of small GTPases that controls intracellular vesicular trafficking in a compartment-specific manner. Mutations in the $R A B 39 B$ gene cause intellectual disability comorbid with autism spectrum disorder and epilepsy, but the impact of RAB39B loss of function on synaptic activity is largely unexplained. Here we show that protein interacting with C-kinase 1 (PICK1) is a downstream effector of GTP-bound RAB39B and that RAB39B-PICK1 controls trafficking from the endoplasmic reticulum to the Golgi and, hence, surface expression of GluA2, a subunit of alpha-amino-3-hydroxy-5-methyl-4-isoxazole propionic acid receptors (AMPARs). The role of AMPARs in synaptic transmission varies depending on the combination of subunits (GluA1, GluA2 and GluA3) they incorporate. RAB39B downregulation in mouse hippocampal neurons skews AMPAR composition towards non GluA2-containing $\mathrm{Ca}^{2+}$-permeable forms and thereby alters synaptic activity, specifically in hippocampal neurons. We posit that the resulting alteration in synaptic function underlies cognitive dysfunction in RAB39B-related disorders.

\footnotetext{
${ }^{1}$ Dulbecco Telethon Institute at IRCCS San Raffaele Scientific Institute, Division of Neuroscience, 20132 Milan, Italy. ${ }^{2}$ F. Hoffmann-La Roche AG, pRED Pharma Research \& Early Development, DTA Neuroscience, CH4070 Basel, Switzerland. ${ }^{3}$ Vita-Salute San Raffaele University, 20132 Milan, Italy.

${ }^{4}$ Department of Life Sciences, University of Modena and Reggio Emilia, 41125 Modena, Italy. ${ }^{5}$ CNR Institute of Neuroscience, Department of BIOMETRA, University of Milan, 20129 Milan, Italy. ${ }^{6}$ Department of Neuroscience and Howard Hughes Medical Institute, Johns Hopkins University School of Medicine, Baltimore, Maryland 21205, USA. ${ }^{7}$ IRCCS San Raffaele Scientific Institute, Division of Genetics and Cell Biology, 20132 Milan, Italy. ${ }^{8}$ Centro de Biología Molecular Severo Ochoa, Consejo Superior de Investigaciones Científicas/Universidad Autónoma de Madrid, Madrid 28049, Spain. * These authors contributed equally to this work. Correspondence and requests for materials should be addressed to P.D. (email: p.dadamo@hsr.it).
} 
T he human $R A B 39 B$ gene, which maps to the distal $\mathrm{Xq} 28$ locus, encodes RAB39B, a member of the RAB GTPases, small monomeric GTPases belonging to the RAS-like GTPase superfamily, that have a key role in the regulation of intracellular vesicular trafficking. Indeed, RAB GTPases are physically associated with specific organelles, through specific effector protein interaction, and act as a network to regulate both spatially and temporally the transport of specific vesicles switching from the active GTP-bound and the inactive GDPbound state ${ }^{2,3}$. RAB and RAB-associated proteins have been shown to play an important role in a number of rare monogenic as well as multifactorial diseases ${ }^{4,5}$ characterized by cognitive deficit. In particular, previous work on X-linked intellectual disability (XLID) has identified mutations in the GDI1 gene that cause loss of function of the RAB-interacting protein $\alpha \mathrm{GDI}^{6,7}$. RAB GTPases and interacting proteins are abundantly expressed in the central nervous system across species, and Gdil mouse models have been relevant to demonstrate the causal link of $\alpha \mathrm{GDI}$ loss of function with deficits in glutamate release and cognitive impairment ${ }^{8,9}$. Our group previously demonstrated that loss-offunction mutations in human $R A B 39 B$ gene are associated with XLID comorbid with autism spectrum disorder and seizures ${ }^{10}$ and also comorbid with early onset of Parkinson's disease ${ }^{11}$. Moreover, it was reported that also a $0.5-\mathrm{Mb}$ tandem copynumber gain at distal Xq28 including $R A B 39 B$ was linked to $\mathrm{XLID}^{12}$. We defined that RAB39B is a neuronal-specific protein localized at the Golgi compartment, but its functional role is unknown ${ }^{10}$. The demonstrated link with a human genetic disease urged us to define the functional role of RAB39B in neurons and the potential link to cognitive function.

In the present study, we define that RAB39B interacts with protein interacting with C-kinase 1 (PICK1), which became an attractive target for further studies. Previous studies defined the functional role of PICK1 in synaptic alpha-amino-3-hydroxy-5methyl-4-isoxazole propionic acid receptor (AMPAR) surface expression, trafficking and post-synaptic targeting ${ }^{13-18}$, but the mechanism of AMPAR biogenesis, assembly and trafficking is poorly understood. In hippocampal CA1 pyramidal neurons, AMPARs are hetero-tetramers formed in the endoplasmic reticulum (ER) ${ }^{19-21}$ mostly composed of GluA1/GluA2 or GluA2/GluA3 hetero-dimers ${ }^{22}$. Export of AMPARs from the $\mathrm{ER}$ requires the interaction of the C-terminal domain of AMPAR subunits with other proteins. In fact, synapse-associated protein 97 (SAP97) interacts with the immature GluA1 C-terminal domain ${ }^{23}$ early in the secretory pathway ${ }^{24}$, while GluA2 C-terminal domain interacts with PICK1, which is necessary for GluA2 exit from the ER. Thus, PICK1 selectively binds GluA2/GluA3 heterodimers for exit from the ER to Golgi compartment ${ }^{25}$.

Given the RAB39B-PICK1 interaction and the Golgi localization of RAB39B, here we hypothesize that RAB39B might be a key molecule fundamental to regulate a specific secretory trafficking step of GluA2 AMPAR subunit. Supporting this idea, $R a b 39 b$-silenced mouse hippocampal neurons show increased GluA2 immature form suggesting ER retention. This result provokes, as a final step, a reduction in the amount of GluA2 AMPAR subunits at the post-synaptic membrane, leading to the formation of GluA2-lacking, $\mathrm{Ca}^{2+}$-permeable AMPARs, often associated with immature synapses and cognitive impairment ${ }^{26}$.

We report here that $\mathrm{RAB} 39 \mathrm{~B}$ is the key molecule regulating the translocation of GluA2/GluA3 heterotetramers into the Golgi. Indeed, in the absence of RAB39B, AMPARs arrangement at the post-synaptic site is misregulated, which provides an explanation for the involvement of $R A B 39 B$ mutations in the aetiology of ID and autism spectrum disorder.

\section{Results}

GTP-bound RAB39B interacts with PICK1. To understand the causal link between XLID and gene defects associated with RAB39B, we set out to map the role of RAB39B in vesicular trafficking. As a first step, we searched for downstream effectors of RAB39B by use of a yeast two-hybrid screening (Supplementary Fig. 1a-c). With the human full-length RAB39B protein as bait, by screening a human fetal brain cDNA library we identified a protein originally described as protein interacting with C-kinase 1 (PICK1) as the strongest interacting molecule. In agreement with our results from the two-hybrid screen, recombinant GST-RAB39B locked in the active state with the nonhydrolysable GTP analogue GTP $\gamma \mathrm{S}$ and the dominant active GST-RAB39B Q68L efficiently pulled down PICK1, as compared with GDP-bound or dominant-negative GST-RAB39B S22N, using whole-brain lysate of murine origin (Fig. 1a,b). Instead, recombinant GST-RAB39A, sharing 76\% identity at protein level with RAB39B, did not pull down PICK1 (Supplementary Fig. 1d).

GST-RAB39B GTP $\gamma$ S likewise pulled down PICK1, obtained by in vitro transcription and translation, which indicated that PICK1 interacts directly with RAB39B, and mostly in its GTPbound state (Fig. 1c). Furthermore, myc-tagged PICK1 coimmunoprecipitates with flag-tagged RAB39B from lysates of HEK293T cells in which the two constructs were co-expressed (Fig. 1d). Finally, we performed the reverse pull down using GSTPICK1 and found that it pulled down RAB39B from the mouse brain lysate, as well as previously identified interacting proteins PKC $\alpha^{27,28}$ and the GluA2 AMPAR subunit ${ }^{29}$ (Fig. 1e).

RAB39B effector-binding region interacts with PICK1 PDZ domain. To define how the two proteins interact at the molecular level, COS7 cells were transfected with RAB39B or PICK1 mutant constructs and interactions were tested again by pull down assays. Since GST-PICK1 did not pull down RAB39B construct lacking a stretch (flag- $\Delta E R$, aa 35-49) corresponding to the canonical effector-binding region as present in all RAB proteins ${ }^{30}$, we concluded that the association between RAB39B and PICK1 is a conventional RAB-effector protein interaction (Fig. 2a). To better define the residues involved in the PICK1-RAB39B effectorbinding region, RAB39B constructs carrying different point mutations were used (flag-D38A, flag-V41G42V43AAA or flagD44F45AA). Such RAB39B constructs were generated based on the predicted structural complex between RAB39B and PICK1 PDZ (see below). Consistently with structure predictions, GSTPICK1 did not pull down RAB39B construct carrying D44 and F45 mutated to alanines (aa D44F45AA), indicating that such amino-acid pair in the $\mathrm{N}$-terminal half of the $\beta 2$-strand of RAB39B play a central role in the association between RAB39B and PICK1 (Fig. 2a). PICK1 is a PDZ- and BAR-domain-containing protein. GST-RAB39B GTP $\gamma S$ pulled down recombinant myc-tagged PDZ domain (myc-PDZ; aa 23-99), but not flagtagged recombinant PICK1 lacking the PDZ domain (flag- $\Delta 121$, aa 1-121; Fig. 2b). Thus, the interface between the two interacting proteins involves amino acids D44 and F45 of the ER domain of RAB39B and, respectively, the PDZ domain of PICK1.

The PDZ domain of PICK1 has been shown before to interact with a variety of proteins ${ }^{31,32}$, and point mutations within the PDZ domain of lysine 83 (K83) or of both lysine 27 (K27) and aspartate 28 (D28) with alanines abolished protein binding $29,31,33,34$. Since GST-RAB39B GTP $\gamma$ S pulled down fulllength myc-PICK with the K83A substitution (myc-K83A), but not with the K27D28AA substitutions (myc-KDAA), we concluded that K27 and D28 in the PDZ domain is key for the interaction of PICK1 with RAB39B (Fig. 2b). 
a
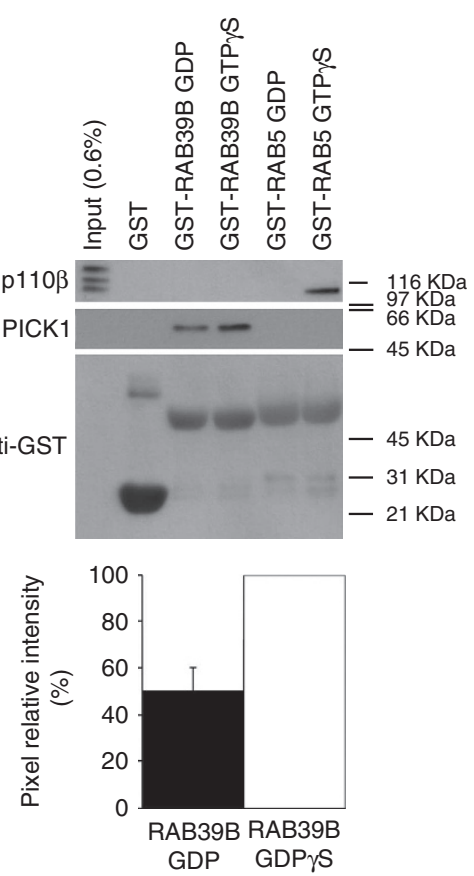

d

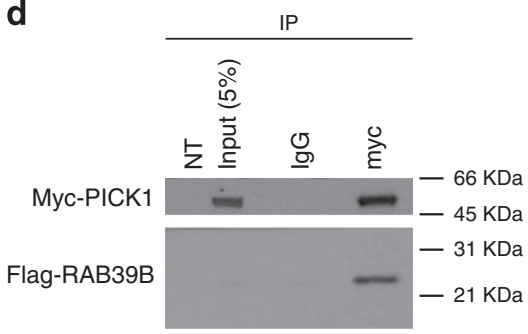

b
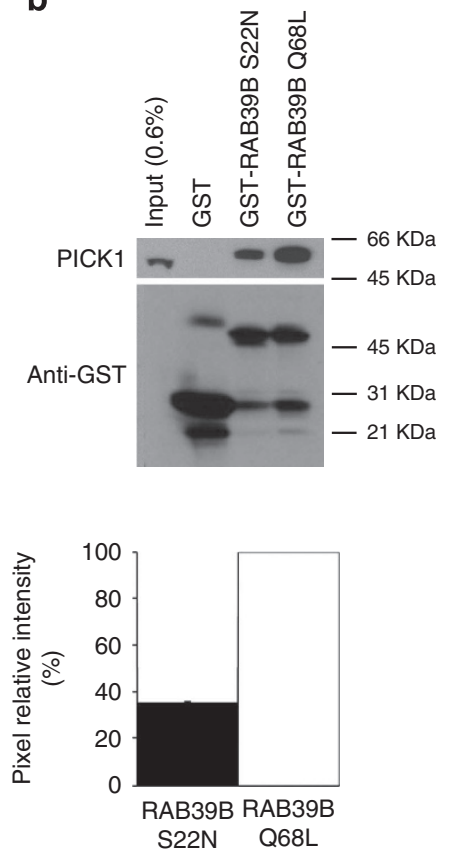

e

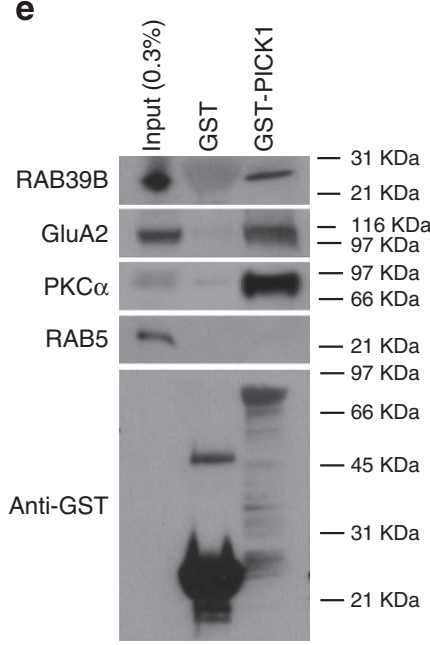

C

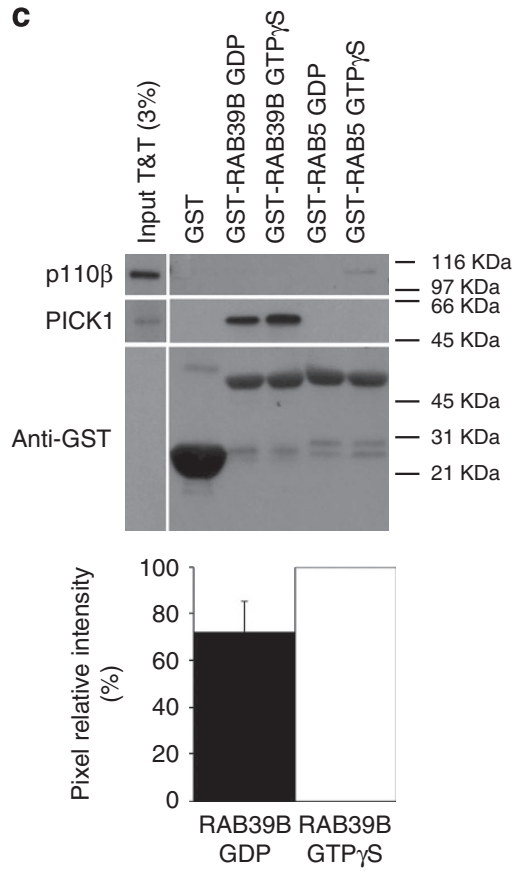

Figure 1 | RAB39B interacts with PICK1. (a-c, upper panel) Representative western blots ( $n=3$ experimental replicates) of GST-RAB39B GDP/GTP $\gamma$ S and GST-RAB39B S22N/Q68L pull down. (a,b) Input: mouse brain lysate. (c) Input T\&T: PICK1 in vitro transcribed and translated. (a-c, lower panel) Histograms show the percentage \pm s.e.m. of PICK1 binding to GST-RAB39B GDP versus GST-RAB39B GTP $\gamma$ S. (d) Representative western blot ( $n=3$ experimental replicates) of co-immunoprecipitation of myc-PICK1 and flag-RAB39B. Input: HEK293T cells; NT: non-transfected cells. (e) Representative western blots ( $n=3$ experimental replicates) of GST-PICK1 pull down. Input: mouse brain lysate.

In agreement with our in vitro binding assays, we co-expressed flag-RAB39B and myc-PICK1 constructs in COS7 cells that lack the corresponding endogenous proteins (Fig. 3). Pearson's correlation coefficient (PCC) analysis on immunofluorescence indicated that RAB39B and PICK1 co-localized at the perinuclear region and at the plasma membrane (flag-RAB39B/myc-PICK1: $n=14$ cells, $\mathrm{PCC}=0.70$; Fig. $3 \mathrm{~b}, \mathrm{~h}$ ). As a control of specificity of RAB39B co-localization with PICK1, we co-expressed GFPRAB39A with myc-PICK1, because RAB39A is endogenously expressed in COS7 cells. PCC analysis revealed that PICK1 highly co-localized with RAB39B but not with RAB39A (GFP-RAB39A/ myc-PICK1: $n=27$ cells, $P C C=0.29$, adjusted $P$-value for multiple comparison $(P$-adj. $)=1 \mathrm{E}-08$; Supplementary Fig. 2a-c). Moreover, PCC from immunofluorescence on cotransfection of the relevant mutant constructs in COS7 cells showed that the co-localization of PICK1 and RAB39B was significantly abolished when either RAB39B is mutated in the D44F45AA (flag-RAB39B D44F45AA/myc-PICK1: $n=14$ cells, $\mathrm{PCC}=0.23, P$-adj. $=1.7 \mathrm{E}-08$; Fig. $3 \mathrm{~g}, \mathrm{~h})$ or PICK1 is mutated in the PDZ domain (flag-RAB39B/myc-PICK1 KDAA: $n=23$ cells, $\mathrm{PCC}=0.22, P$-adj. $=1.7 \mathrm{E}-08$; Fig. $3 \mathrm{c}, \mathrm{h})$.

RAB39B-PICK1 interaction is necessary and sufficient for GluA2 trafficking. Given that RAB proteins direct vesicular transport $^{2}$ and previous studies defined the functional role of PICK1 in GluA2 but not GluA1 AMPAR subunit trafficking ${ }^{14,25,35}$, we hypothesized that based on RAB39B and PICK1 interaction, they jointly coordinate trafficking of GluA2, but not of GluA1. To test these assumptions, we transfected into 

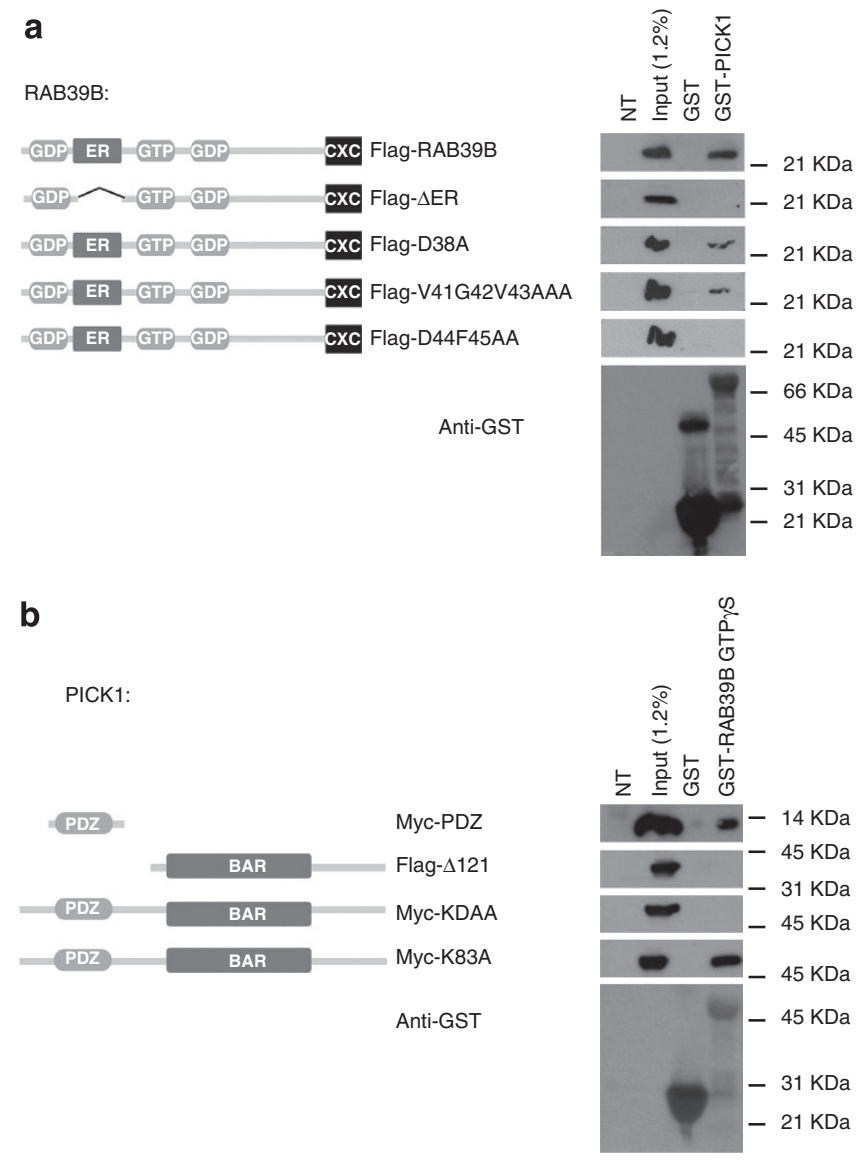

Figure 2 | RAB39B-PICK1 interacting domains. Representative western blots ( $n=3$ experimental replicates) of GST-pull down using (a) GST-PICK1 and (b) GST-RAB39B GTP $\gamma$ S. Input: COS7 cells transfected with differently tagged RAB39B and PICK1 constructs. NT: non-transfected cells.

the heterologous COS7 cell system tagged constructs of RAB39B, PICK1, GluA1 and GluA2 either individually or in combination. Pearson's correlation coefficient was measured to verify colocalization between these proteins (Fig. 4) and the receptor trafficking to the plasma membrane by total internal reflection fluorescence (TIRF) (Fig. 5).

When expressed alone, GFP-GluA1 as well as GFP-GluA2 localized at the perinuclear region (Fig. 4a). Co-expression of flagRAB39B did not change the localization of either GFP-GluA1 or GFP-GluA2, neither RAB39B co-localized with GluA1 and GluA2 (flag-RAB39B/GFP-GluA1: $n=26$ cells, $P C C=0.09$, flag-RAB39B/GFP-GluA2: $n=26$ cells, $P C C=0.06$; Fig. 4c,d,i). Co-expression of myc-PICK1 and GFP-GluA1 did not show co-localization (myc-PICK1/GFP-GluA1: $n=29$ cells, PCC = -0.01 ; Fig. 4e,i). Conversely, GFP-GluA2 co-clustered with myc-PICK at the perinuclear region when co-expressed, as has been previously reported $^{29}$ (myc-PICK1/GFP-GluA2: $n=38$ cells, PCC $=0.66$; Fig. 4f,i).

Remarkably, GFP-GluA2 co-localized with flag-RAB39B only when co-expressed with myc-PICK1, and a significant correlation was found between RAB39B and GluA2 depending on the presence of PICK1 in the cells (flag-RAB39B/GFP-GluA2 with PICK1: $n=12$ cells, $\mathrm{PCC}=0.71, P$-adj. $=1.8 \mathrm{E}-08$ comparing flag-RAB39B/GFP-GluA2 in PICK1 absence versus presence; Fig. 4h,i). In contrast, GFP-GluA1 never showed co-localization regardless of the co-expression of RAB39B and PICK1 constructs (flag-RAB39B/GFP-GluA1 with PICK1: $n=24$ cells, $\mathrm{PCC}=0.06$; Fig. 4g,i).
Next, we then demonstrated receptor trafficking to the plasma membrane by TIRF. Co-expression of flag-RAB39B did not drive the plasma membrane translocation of either GFP-GluA1 or GFP-GluA2 (Fig. 5a,b). Remarkably, in triple transfected cells, flag-RAB39B mediated the GFP-GluA2 translocation to the plasma membrane in the presence of myc-PICK1 (Fig. 5d) but not GFP-GluA1 (Fig. 5c).

All together our results clearly indicate that the effectorbinding region of RAB39B interacts with the PDZ domain of PICK1, also required for interaction with the GluA2 AMPAR subunit $^{36}$. We also demonstrated that the RAB39B-PICK1 interaction is necessary for the RAB39B-driven trafficking of GluA2 cargo. PICK1-mediated functional linkage between RAB39B and GluA2-containing AMPAR relies on PICK1 dimer to act as a scaffold that hosts the other two proteins. In fact, GluA2 co-immunoprecipitates with PICK1 and RAB39B in a wild -type mouse brain lysate, but not in the Pick1-knockout brain, demonstrating the existence of the endogenous complex and that PICK1 acts as the bridge between RAB39B and GluA2 (Fig. 5e).

A model of interactions among the RAB39B-PICK1-GluA2. To gain insight, at the atomic level, into the supramolecular organization of RAB39B, PICK1 and GluA2 AMPAR subunit, we modelled the structure of RAB39B:2PICK1:GluA2 complex (Fig. 6a and Supplementary Methods). This required comparative modelling and rigid-body docking to predict structure and interaction modes of PICK1 and RAB39B (see Methods). In contrast, the complex between the GluA2 C-terminus (GluA2Ct; aa 858-ESVKI-862) and PICK1 was extracted from a conformational ensemble solved by $\mathrm{NMR}^{37}$. In our structural model, the $\mathrm{N}$-terminal half of the $\beta 2$-strand of RAB39B (that is, amino-acid stretch 43-47 of the effector-binding region) interacts with the PDZ domain of PICK1 in a similar and partially overlapping manner as GluA2Ct, which is a $\beta$-strand as well (Fig. 6a-c). The major portions of RAB39B participating in the interface with PICK1 PDZ include (a) the 43-47 segment of the $\beta 2$-strand, which makes inter-backbone and inter-side chain interactions with PICK1 $\beta \mathrm{B}$ and with $\alpha \mathrm{B}$; (b) switch 1 (swI) that interacts with the PICK1 $\beta \mathrm{A} / \beta \mathrm{B}$ loop and $\alpha \mathrm{B}$; and (c) the switch 2 (swII) that interacts with PICK $1 \beta C$ as well as the $\beta \mathrm{A} / \beta \mathrm{B}$ and $\beta \mathrm{B} / \alpha \mathrm{A}$ loops $(\mathrm{b}$ and $c)$. The predicted complex highlights D44 and F45 in the $\mathrm{N}$-terminal half of the $\beta 2$-strand as playing a central role in the interface, D44 making a salt bridge with K83 of PICK1 PDZ. Such a centrality of $\mathrm{D} 44$ and $\mathrm{F} 45$ has been validated by in vitro experiments (Fig. 3). Remarkably, the PICK1 $\beta A / \beta B$ loop of PDZ holds the K27-D28 pair that we found essential for PICK1 - RAB39B interaction. Structure predictions indicate D28 as more important than $\mathrm{K} 27$ in $\mathrm{RAB} 39 \mathrm{~B}$ recognition. Indeed, K27 is buried at the $\beta \mathrm{B} / \alpha \mathrm{B}$ interface, its positively charged nitrogen atom being about $9 \AA$ from the carboxylate oxygen atoms of D38 on the swI of RAB39B, and we postulate that the effect of the K27A mutation on RAB39B recognition, if any, is indirect. In contrast, D28 recognizes the $\mathrm{G}$ protein via a salt bridge with $\mathrm{R} 70$ in swII. Structure prediction therefore suggests that the interactions of RAB39B and GluA2 with the PDZ domain of PICK1 are mutually exclusive. In line with evidence that PICK1 can dimerize ${ }^{33}$, the structural model suggests that dimerization of PICK1 is a prerequisite for simultaneous recognition of both RAB39B and GluA2 each by one of the PICK1 molecules in the PICK1 dimer (Fig. 6a-c). The existence of such complex is supported by our co-immunoprecipitation experiments shown above.

RAB39B directs GluA2 trafficking in neurons. The two most prevalent AMPAR variants contain a tandem of either GluA1 and 

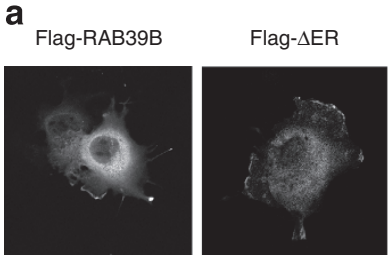

Flag-D38A

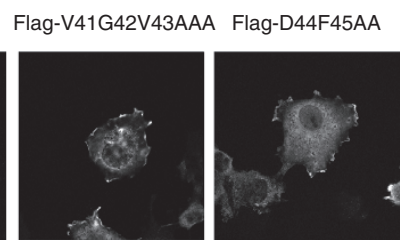

Myc-PICK1

Myc-KDAA
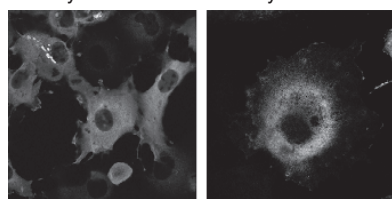

b

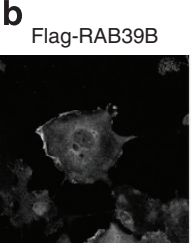

Myc-PICK1
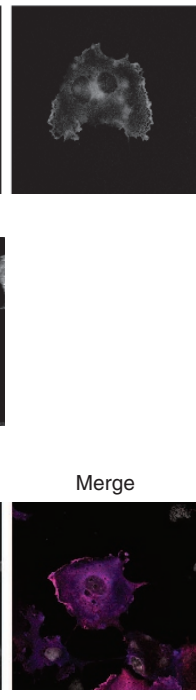

d

Flag- $\Delta$ ER

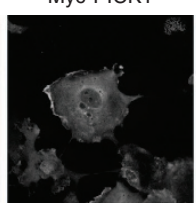

c

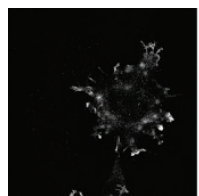

Myc-PICK1 Merge
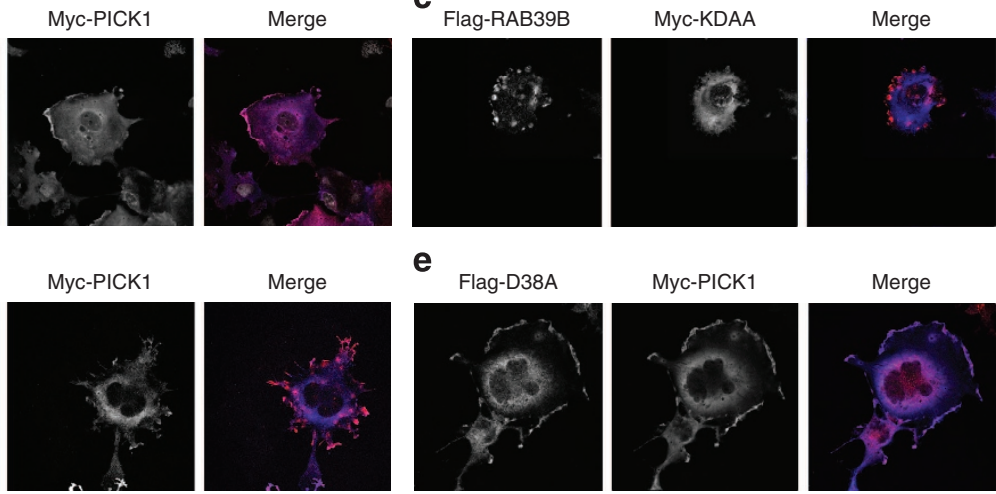

e

Flag-D38A
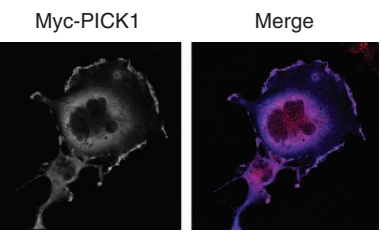

$\mathbf{f}$

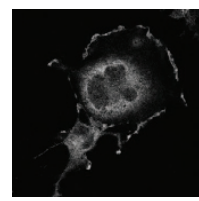

g

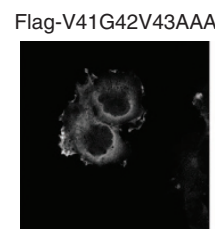

Myc-PICK1

Merge
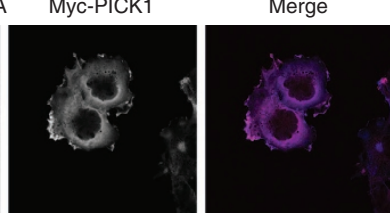

Flag-D44F45AA
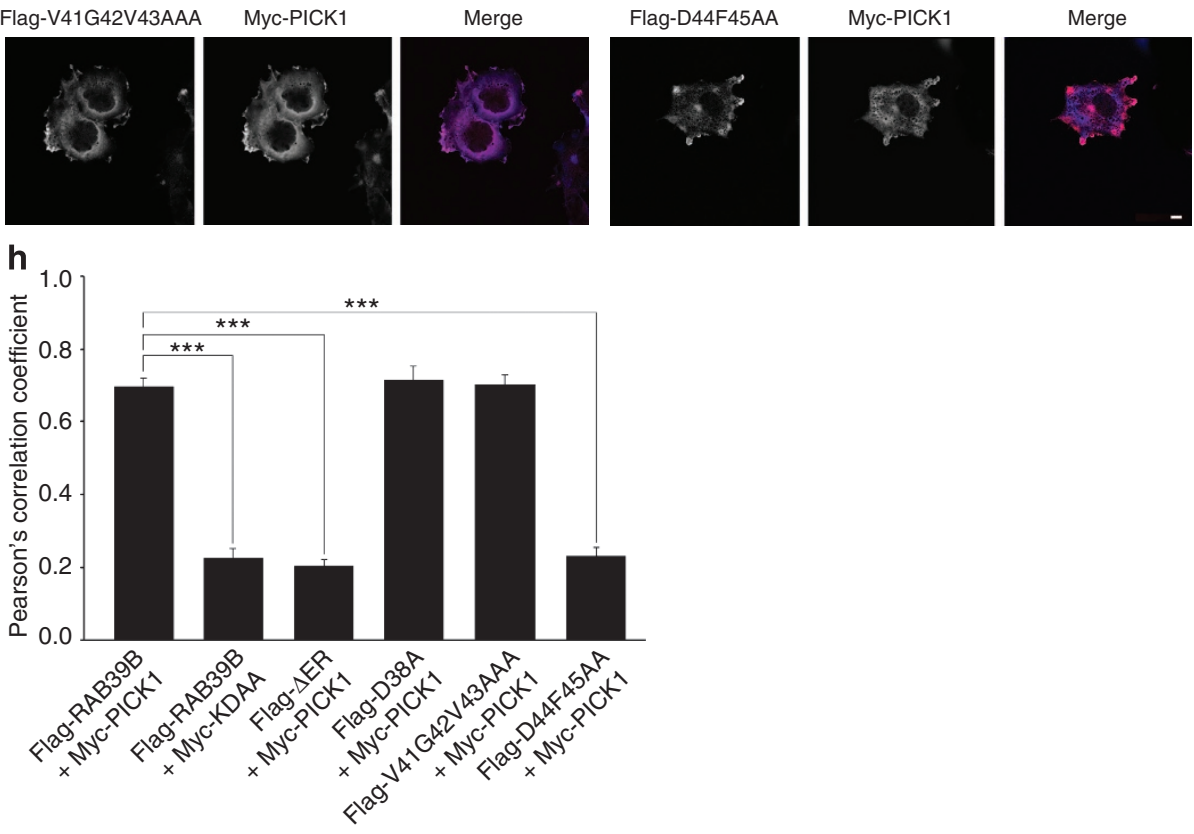

Figure 3 | Validation of RAB39B-PICK1 interaction in COS7 cells. Representative immunofluorescence images of COS7 cells (a) single or (b-g) double transfected with different RAB39B (flag-RAB39B, red) and/or PICK1 (myc-PICK1, blue) constructs. Scale bar, $10 \mu \mathrm{m}$. (h) Histogram shows the Pearson's correlation coefficients (PCC; means \pm s.e.m.) for each co-transfection. Significant statistical differences were found comparing flag-RAB39B/myc-PICK1 PCC $(n=14$ cells; 3 experimental replicates; PCC $=0.70)$ to flag-RAB39B/myc-KDAA PCC $(n=23$ cells; three experimental replicates; PCC $=0.22$, $P$ adj. $=1.7 \mathrm{E}-08)$, to flag- $\Delta \mathrm{ER} / \mathrm{myc}-\mathrm{PICK1} \mathrm{PCC}(n=27$ cells; three experimental replicates; $P C C=0.2, P$ adj. $=1.7 \mathrm{E}-08)$, and to flag-D44F45AA/mycPICK1 PCC ( $n=14$ cells; 3 experimental replicates; PCC $=0.23, P$ adj. $=1.7 \mathrm{E}-08)$. Benjamini-Hochberg procedure used to test statistical significance. ${ }^{\star \star \star \star} P<0.001$.

GluA2 or GluA2 and GluA3 subunits ${ }^{19-21}$. Exports of GluA1/ GluA2 hetero-tetramers from the ER requires GluA1 interaction with SAP97 (refs 23,24), while GluA2/GluA3 hetero-tetramers need GluA2 interaction with PICK1 (ref. 25).
To assess whether the RAB39B-PICK1 tandem directs trafficking of GluA2/GluA3 hetero-tetramers in their endogenous neuronal context, we first analysed the intracellular localization of RAB39B and PICK1 in flag-RAB39B-transfected primary mouse 
a

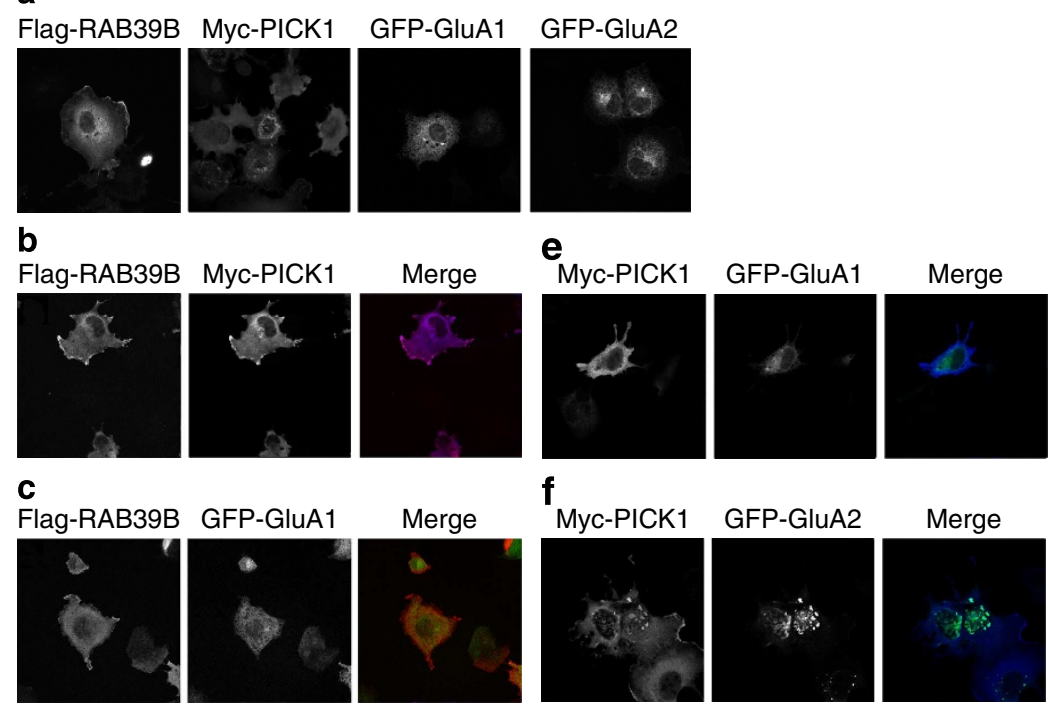

d
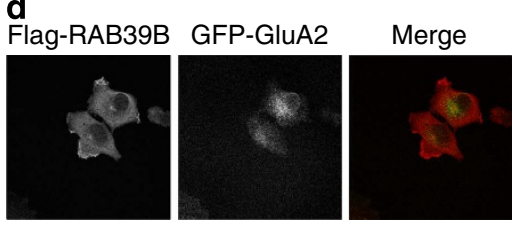

g
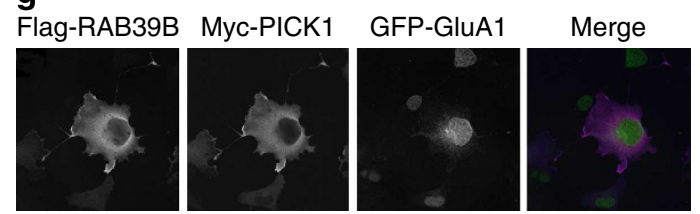

h

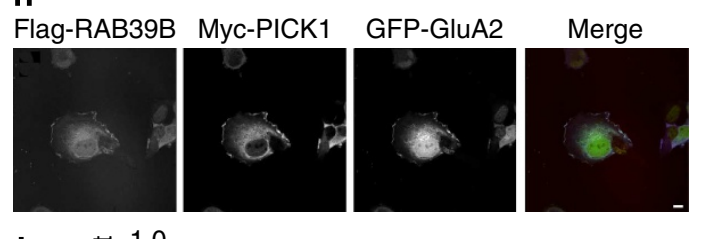

i

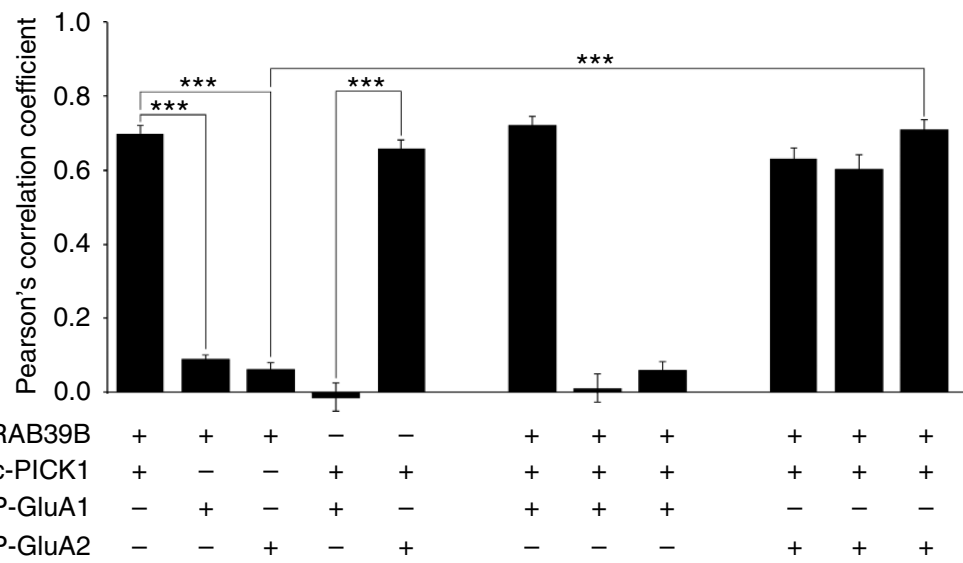

Figure 4 | RAB39B localizes with GluA2 only in the presence of PICK1. Representative immunofluorescence images of COS7 cells (a) single, (b-f) double or (g,h) triple transfected with flag-RAB39B (red), myc-PICK1 (blue), GFP-GluA1 (green) or GFP-GluA2 (green). Scale bar represents $10 \mu$ m. (i) Histogram shows the Pearson's correlation coefficients (PCC; means \pm s.e.m.) for each transfection. PCC was calculated between proteins highlighted with a bold ' + '. Significant statistical differences were found comparing: flag-RAB39B/GFP-GluA1 PCC $(n=26$ cells; three experimental replicates; PCC $=0.09, P$ adj. $=1.8 \mathrm{E}-08)$ and flag-RAB39B/GFP-GluA2 PCC $(n=22$ cells; three experimental replicates; $P C C=0.06, P$ adj. $=1.8 \mathrm{E}-08)$ to flag-RAB39B $/ \mathrm{myc}-$ PICK1 PCC $(n=14$ cells; three experimental replicates; PCC $=0.70)$; myc-PICK1/GFP-GluA1 PCC $(n=29$ cells; three experimental replicates; PCC $=-0.01, P$ adj. $=1.8 \mathrm{E}-08)$ to myc-PICK1/GFP-GluA2 PCC ( $n=38$ cells; three experimental replicates; PCC $=0.66) ;$ flag-RAB39B/GFP-GluA2 PCC in the absence or presence of PICK1 (PCC in the presence of PICK1 $=0.71 ; n=12$ cells; three experimental replicates; $P$ adj. $=1.8 \mathrm{E}-08$ ). BenjaminiHochberg procedure used to test statistical significance. ${ }^{\star \star \star} P<0.001$. 


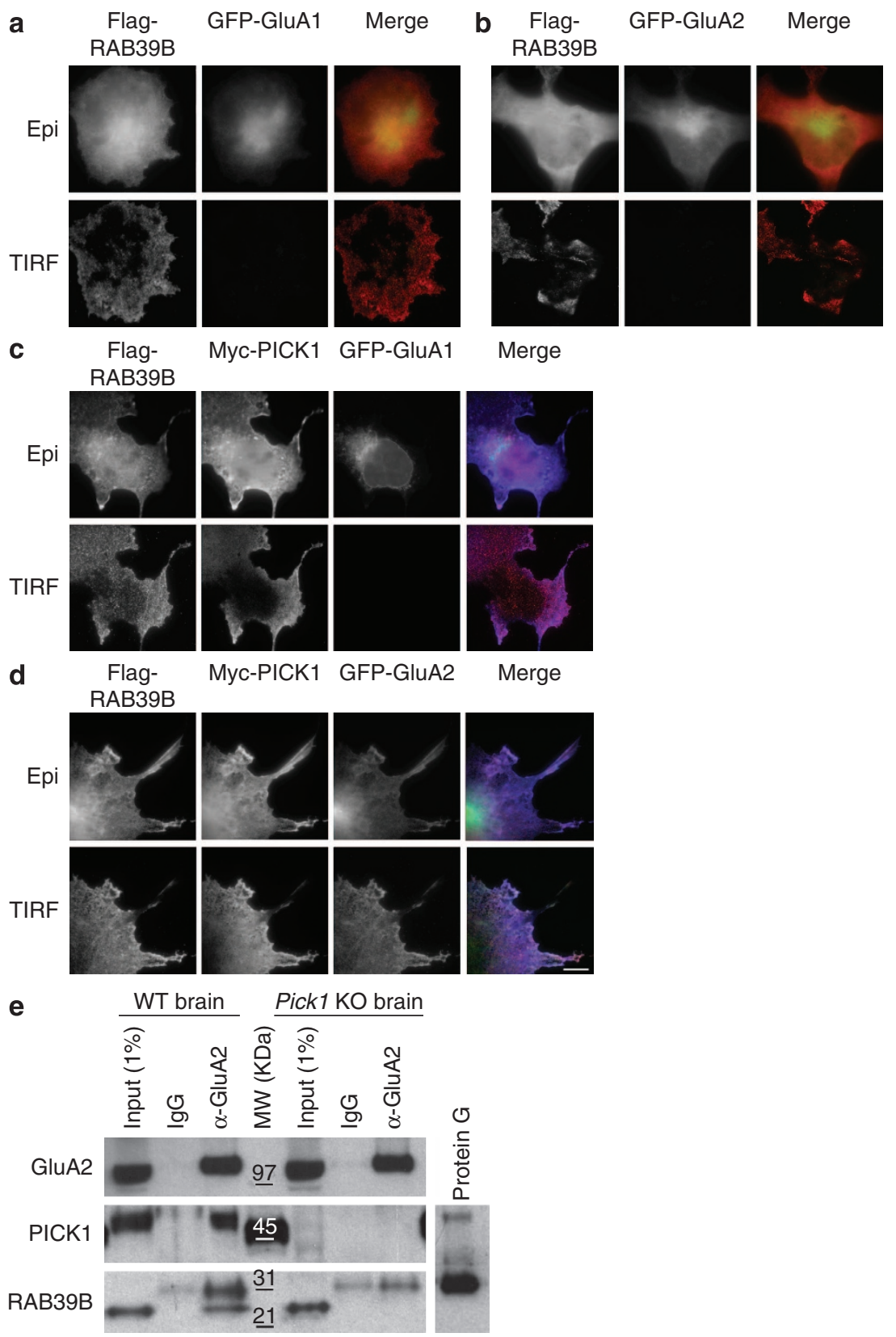

Figure 5 | Validation of RAB39B-PICK1-GluA2 complex. Representative TIRF and epifluorescence images of COS7 cells transfected with flag-RAB39B (red) and GFP-GluA1 (green) or GFP-GluA2 (green), in the absence (a-b) or presence (c,d) of PICK1 (blue). Scale bar, $10 \mu \mathrm{m}$. (e) Representative western blots ( $n=3$ experimental replicates) of immunoprecipitation from WT and Pick1 KO mouse brain lysates (Input). The 31-kDa contaminant bands reacting with the anti-RAB39B correspond to the protein $\mathrm{G}$ as shown in the mock elution of protein $\mathrm{G}$ Sepharose-4 fast flow beads (in conditions of elution adopted as described in Methods ${ }^{69}$ ).

hippocampal neurons (Supplementary Fig. 3). Flag-RAB39B appears to partially co-localize with PICK1 in the cell body, instead of along dendrites (Supplementary Fig. 3a). In a similar manner, flag-RAB39B and PICK1 co-localize in part with the ER or Golgi markers, calreticulin and GM130, respectively (Supplementary Fig. 3b,c). No co-localization was observed between flag-RAB39B and adaptor protein 2 (AP2), which was used as a negative control ${ }^{10}$. Although RAB39B and PICK1 are certainly involved in many other intracellular pathways, our data support that RAB39B-PICK1 interaction has a role in cargo trafficking between the ER and the Golgi compartments.

We then analyse the effect of Rab39b downregulation in transduced murine primary hippocampal neurons with previously described shRab39b and shScramble lentiviral particles ${ }^{10}$. Silencing with shRab39b led to a $40 \%$ downregulation of RAB39B expression levels (mean \pm s.e.m., shScramble: $1.1 \pm 0.13$, shRab39b: $0.68 \pm 0.08 ; n=3$; $t$-test $P=0.035$ ), without affecting protein expression levels of PICK1, GluA1, GluA2 and GluA3 (Supplementary Fig. 4).

We first determined the effect of RAB39B silencing on the intracellular localization of PICK1 GluA1, GluA2 and GluA3 in the murine hippocampal neurons by immunofluorescence at 14 DIV. We found an increase in PICK1, GluA2 and GluA3 levels in the cell body of shRab39b-treated neurons as compared with the neurons treated with the control shRNA (mean \pm s.e.m., PICK1 shScramble $n=21: 2.98 \pm 0.28$, shRab39b $n=21: 4.25 \pm 0.35$; 
a
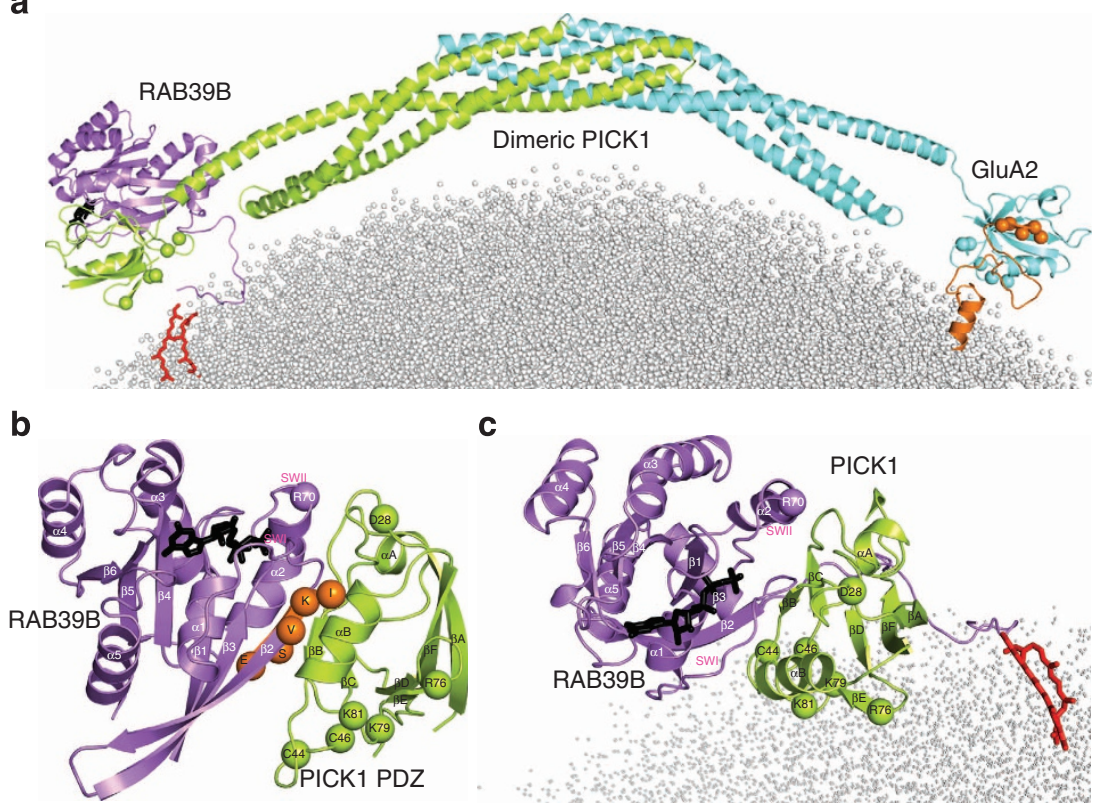

C

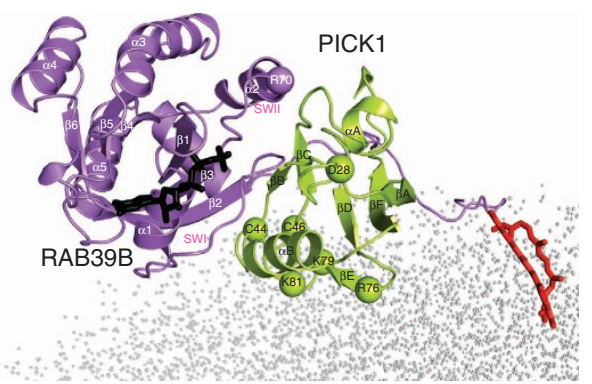

Figure 6 | Dimeric PICK1 acts as a scaffold for RAB39B and GluA2. (a) A zoom into the predicted RAB39B:2PICK1:GluA2Ct complex is shown on a liposome. (b,c) A zoom into the PICK1 PDZ-RAB39B interface is shown. The GluA2Ct extracted from the other PDZ subunit, following the C $\alpha$-atom superimposition of the two PDZ domains, is shown, represented as orange spheres. The spheres of the interacting D28 from PICK1 PDZ and R70 from RAB39B are shown as well. (c) An alternative view of the PICK1 PDZ-RAB39B complex is shown highlighting the orientation of the lipid-binding motifs on PDZ and of the two geranylgeranyl molecules on the two C-terminal cysteines of RAB39B with respect to the surface of the liposome.

$t$-test $\quad P=0.009 ; \quad$ GluA2 shScramble $\quad n=9: \quad 0.60 \pm 0.05$, shRab39b $n=5$ : $0.88 \pm 0.05$; $t$-test $P=0.003$; GluA3 shScramble $n=6: 0.42 \pm 0.03$, shRab39b $n=7: 0.62 \pm 0.04 ; t$-test $P=0.005$; Fig. $7 \mathrm{a}$ and Supplementary Fig. 5a), which suggests that there is a defect in protein trafficking when levels of RAB39B are downregulated.

Next, we analysed the effect of RAB39B silencing on the intracellular trafficking in murine hippocampal neurons of the AMPAR subunits GluA1, GluA2 and GuA3 by examining their glycosylation status. Both AMPAR subunits are $N$-glycosylated and their $\mathrm{N}$-glycans are subsequently modified in the ER before they are targeted to the plasma membrane. We therefore deglycosylated lysates from RAB39B silenced or control neurons with either Endo- $\beta$ - $N$-acetylglucosaminidase $\mathrm{H}$ (EndoH), which removes only unmodified $\mathrm{N}$-glycans ${ }^{25}$, or, as a control, with peptide- $N$-Glycosidase F (PNGasef), which removes all $\mathrm{N}$-glycans, before analysis of the AMPAR subunits by immunoblotting. In the control lysates two isoforms, differing in their mobility in gel, were detected for GluA1, GluA2 and GluA3 on treatment with EndoH: an upper band, corresponding to the mature isoform (as it acquired partial EndoH resistance), and a lower band, corresponding to the immature isoform (as it acquired no EndoH resistance). The ratio between the two bands thus is a measure for AMPAR subunit maturation. As such, we determined that GluA2 and GluA3 maturation was impaired in shRab39b-treated neurons compared with the control neurons (mean \pm s.e.m., GluA2 shScramble: $0.96 \pm 0.05$, shRab39b: $0.52 \pm 0.05 ; \quad n=3 ; \quad t$-test $P=0.002 ; \quad$ GluA3 shScramble: $1.0 \pm 0.07$, shRab39b: $0.0 \pm 0.0 ; n=3$; $t$-test $P=0.0001$; Fig. $7 \mathrm{~b}$ ), while GluA1 maturation was not affected by the shRab39b silencing (mean \pm s.e.m., shScramble: $1.29 \pm 0.21$, shRab39b: $1.28 \pm 0.15 ; n=3$; $t$-test $P=0.9$; Fig. $7 \mathrm{~b})$.

We next examined the role of the RAB39B-PICK1 tandem in post-Golgi trafficking of AMPAR subunits along dendrites. We noted that, in RAB39B-silenced cells, the increased PICK1 as well as GluA2 and GluA3 density at the cell body (Fig. 7a and
Supplementary Fig. 5a) was mirrored by a decreased PICK1, GluA2 and GluA3 density along dendrites (mean \pm s.e.m., PICK1 shScramble $n=14: 17.82 \pm 1.13$, shRab39b $n=15: 14.98 \pm 0.61$; $t$-test $P=0.03$; GluA2 shScramble $n=10$ : $0.82 \pm 0.02$, shRab39b $n=5: 0.49 \pm 0.02$; $t$-test $P=2.8 \mathrm{E}-7$; GluA3 shScramble $n=7$ : $1.24 \pm 0.06$, shRab39b $n=10: 0.62 \pm 0.07 ; \quad t$-test $P=1.6 \mathrm{E}-5$; Fig. 7c and Supplementary Fig. 5b). Likewise, downregulation of RAB39B resulted in a decrease in surface density of GluA2 as measured by immunostaining without permeabilization of the cells (mean \pm s.e.m., GluA2 shScramble $n=89$ : $0.88 \pm 0.02$, shRab39b $n=70$ : $0.72 \pm 0.03$; $t$-test $P=0.006$ ). Curiously, surface expression of GluA1 slightly increased on RAB39B silencing (mean \pm s.e.m., shScramble $n=41: 0.62 \pm 0.02$, shRab39b $n=40$ : $0.77 \pm 0.02$; $t$-test $P=0.03$; Fig. $7 \mathrm{~d}$ ), mirrored by a significant increase along dendrites (mean \pm s.e.m., GluA1 shScramble $n=10: \quad 0.87 \pm 0.02, \quad$ shRab39b $\quad n=10: \quad 1.05 \pm 0.04 ; \quad t$-test $P=0.0007$; Fig. $7 \mathrm{c}$ and Supplementary Fig. 5b). The reintroduction of RAB39B by co-transducing shRab39b-treated neurons with a cherryRab39b-rescue lentiviral particles recovered the intracellular distribution of PICK1 (shRab39b versus Rab39brescue $n=8$; $t$-test $P=0.002$ ), GluA2 (shRab39b versus Rab39brescue $n=11$; $t$-test $P=0.004$ ) and GluA3 (shRab39b versus Rab39b-rescue $n=12$; $t$-test $P=0.007$ ) and GluA1 (shRab39b versus Rab39b-rescue $n=10 ; t$-test $P=0.006)$ and GluA2 (shRab39b versus Rab39b-rescue $n=8$; $t$-test $P=0.01$ ) surface expression (Fig. 7a,c,d and Supplementary Fig. 5a,b).

Finally, downregulation of RAB39B has a mild effect on spine morphology without altering spine number and type (mean $\pm^{-}$ s.e.m., spine length shScramble $n=26: 1.20 \pm 0.02$, shRab39b $n=30: \quad 1.12 \pm 0.02 ; \quad t$-test $P=0.05$; spine width shScramble $n=25: \quad 0.68 \pm 0.01, \quad$ shRab39b $\quad n=29: \quad 0.64 \pm 0.009 ; \quad t$-test $P=0.02$; Supplementary Fig. 5 c).

These results indicate that in neurons the RAB39B-PICK1 tandem directs trafficking specifically of GluA2/GluA3 heterotetramers to the Golgi and, consequently, regulates GluA2 cell surface expression and spine morphology. 
RAB39B determines AMPAR composition and synaptic transmission. Since our results so far demonstrated that RAB39B is key to ensure efficient trafficking of GluA2/GluA3 to the Golgi compartment, and, hence, its cell surface expression, we reasoned that ultimately RAB39B controls the availability of GluA2 and thus AMPAR composition at the post-synapses. Because the subunit composition, in turn, determines $\mathrm{Ca}^{2+}$ permeability of the AMPARs and therefore impacts on synaptic transmission, we asked whether RAB39B-PICK-directed trafficking of GluA2/ GluA3 is key for glutamatergic synapses. To this end, we performed electrophysiological recordings on primary murine hippocampal neurons that were infected with the shRab39b or control shScramble lentiviruses at low doses to ensure that most pre-synaptic inputs would come from non-infected (unaltered)

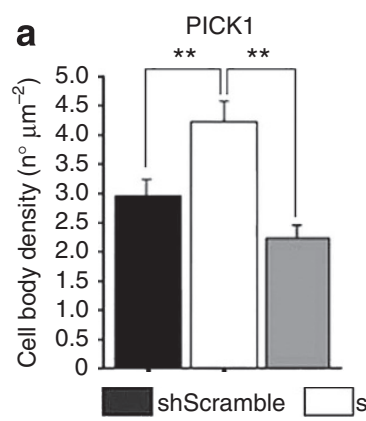

GluA1

GluA2

GluA3
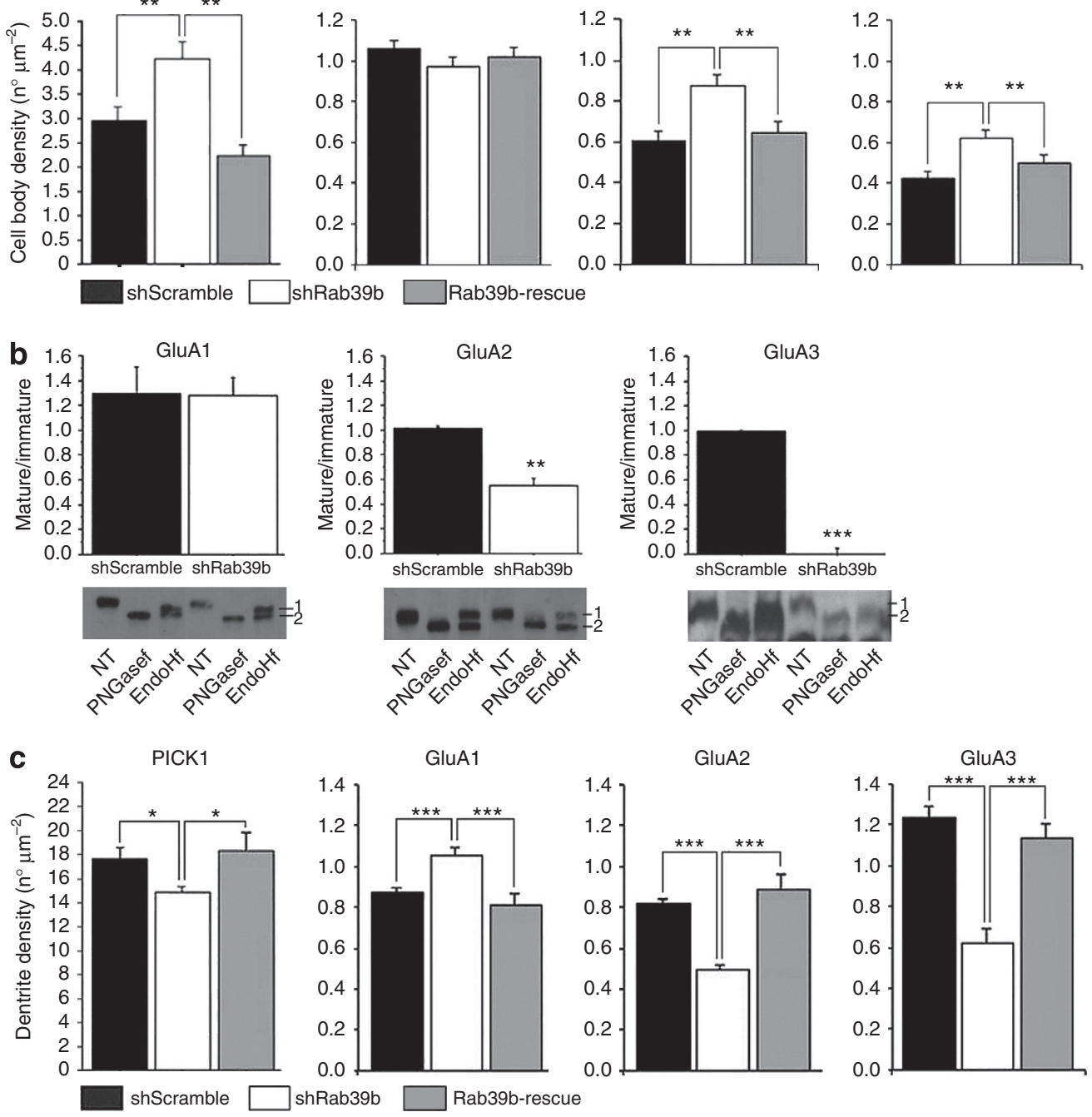

d
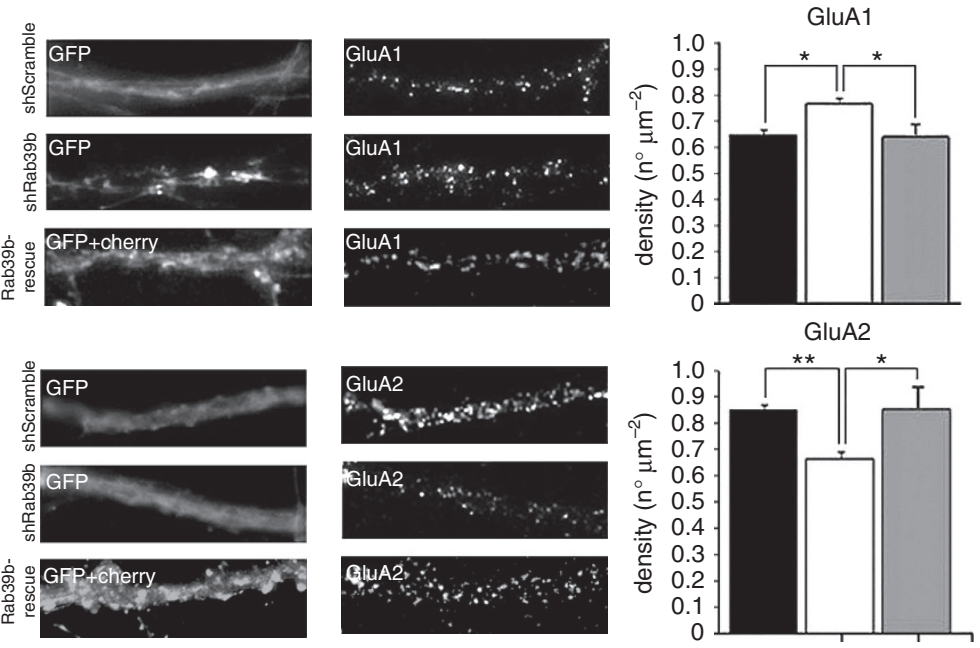

shScramble

shRab39b

Rab39b-rescue 
neurons, while post-synaptic outputs would come from infected (silenced) neurons (Supplementary Fig. 6a,b). The amplitude of miniature EPSCs was slightly but significantly larger in RAB39Bsilenced neurons (mean \pm s.e.m. in $\mathrm{pA}$, shScramble $n=14$ : $-17.1 \pm 1.1$, shRab39b $n=13: \quad-22.9 \pm 1.9 ; \quad t$-test $P<0.01)$ (Fig. $8 \mathrm{a}-\mathrm{c}$ ). The miniature frequency, however, was unaffected, suggesting that indeed RAB39B silencing occurred mostly in post-synaptic neurons due to the low-density lentiviral infection (Fig. 8c). Interestingly, kinetic analysis of the miniature currents indicated that RAB39B silencing accelerated decay (mean \pm s.e.m. in $\mathrm{ms}$, shScramble $n=14: \quad 2.60 \pm 0.32, \quad$ shRab39b $n=13$ : $1.71 \pm 0.05$; $t$-test $P<0.01)$ but not rise times of synaptic currents (Fig. 8b,c). The specificity of shRab39b effect was determined using a rescue experiment. Electrophysiological recordings were carried out on primary murine hippocampal neurons that were infected with the shRab39b or control shScramble lentiviruses at multiplicity of infection (MOI) 1 to ensure that pre-synaptic inputs and post-synaptic outputs come from infected (silenced) neurons and after 6 days in culture transfected with a flagRAB39B-rescue, as previously described ${ }^{10}$ (Supplementary Fig. $6 c, d)$. The reintroduction of RAB39B recovered the amplitude and kinetic of the miniature currents (Fig. $8 \mathrm{~d}-\mathrm{g}$ ).

Since RAB39B directs GluA2 traffic and thus cell surface expression, we attributed the electrophysiological changes on RAB39B silencing to an altered subunit composition of synaptic AMPARs. To test this hypothesis, we monitored inward rectification of AMPAR-mediated synaptic responses in organotypic hippocampal slices from mice that were injected in the CA1 with the relevant lentiviruses (shScramble or shRab39b) by evoked EPSCs, recorded at $-60 \mathrm{mV}$ and $+40 \mathrm{mV}$. The ratio of synaptic responses at $+40 \mathrm{mV}$ over responses at $-60 \mathrm{mV}$ was calculated to serve as a rectification index (R.I.). We found a significant increase in the R.I. of RAB39B silenced neurons (mean \pm s.e.m., uninfected $n=6: \quad 1.801 \pm 0.175$; shScramble $n=6: \quad 1.403 \pm 0.166$; shRab39b $n=6: 2.998 \pm 0.297$; ANOVA: $P<0.0001$ ) (Fig. 8h,i), indicative of enrichment at synapses of inward rectifying AMPARs. Since GluA2-lacking AMPARs in fact are inward rectifying ${ }^{38}$ we concluded that the alterations in synaptic transmission are due to a lowered GluA2 availability. This interpretation also fits with our observation that RAB39B down regulation leads to increased miniature EPSC amplitude (Fig. 8a-g), in agreement with the higher conductance of GluA2lacking AMPARs ${ }^{39}$. Finally, we tested whether long-term depression (LTD) was altered in RAB39B down regulated cells, given the involvement of PICK1 in activity-dependent AMPAR endocytosis and $\mathrm{LTD}^{15,40}$. To this end, we assessed NMDARdependent LTD in shRab39b-, shScramble- and non-infected CA1 neurons (Fig. 8j). After LTD induction (500 stimuli at $1 \mathrm{~Hz}$, with postsynaptic depolarization at $-40 \mathrm{mV}$ ), AMPAR-mediated synaptic responses were equally depressed in the three conditions. These results suggest that, in contrast with PICK1, RAB39B is not required for LTD. Therefore, RAB39B does not appear to be involved in activity-dependent AMPAR endocytosis or recycling.

\section{Discussion}

Our results define RAB39B in tandem with its downstream effector PICK1 as a key molecule dedicated to GluA2 AMPAR subunit trafficking. We demonstrate a molecular complex where the dimerization of PICK1 is a prerequisite for simultaneous recognition of both $\mathrm{RAB} 39 \mathrm{~B}$ and GluA2 by each one of the PDZ domains of PICK1, in a PICK1 dimer conformation.

Subsequently, the remarkable inference from this study is that RAB39B interacting with PICK1 ensures selectively the GluA2/ GluA3 AMPAR exit from the ER and its maturation entering into the cis-Golgi (Fig. 9). Indeed, despite the demonstrated relevance of regulators of AMPAR surface expression, trafficking and postsynaptic targeting and human memory formation and maintenance (for example, KIBRA ${ }^{41}$ and TMS4SF2 (ref. 42)), only few proteins that are involved in the trafficking between the ER and the Golgi of AMPAR subunits are known. Exit of plasma membrane proteins from the ER poses a rate-limiting step and is subjected to tight control, to safeguard that only properly folded and assembled complexes leave the ER and are transported to the cis-Golgi ${ }^{43,44}$, to finally reach the plasma membrane. However, rules governing subunit assembly and the progression of distinct AMPAR complexes through the secretory pathway are largely unknown. It is known that AMPAR subunits preferentially homodimerize soon after the translocation in the ER and tetramerize before ER exit in GluA1/GluA2 and GluA2/GluA3 complexes $^{25}$. Greger and coauthors ${ }^{25}$ found unexpected differences in the subcellular localization and ER export kinetics of the GluA1 and GluA2 subunits. Whereas GluA1 receptor exit the ER and traffic to the surface, GluA2 partly remains in the ER, suggesting the existence of an intracellular GluA2 reserve pool. GluA2 ER pool is, at least partly, associated with GluA3, whereas

Figure 7 | PICK1, AMPARs neuronal localization, maturation and GluA2 surface expression. (a) Quantification of PICK1 (shScramble $n=21$ cells; shRab39b $n=21$ cells; Rab39b-rescue $n=8$ cells; Student's $t$-test shScramble versus shRab39b $P=0.009$; Rab39b-rescue versus shRab39b $P=0.002$ ), GluA1 (shScramble $n=10$ cells; shRab39b $n=10$ cells; Rab39b-rescue $n=9$ cells), GluA2 (shScramble $n=9$ cells; shRab39b $n=5$ cells; Rab39b-rescue $n=11$ cells; Student's $t$-test shScramble versus shRab39b $P=0.003$; Rab39b-rescue versus shRab39b $P=0.004$ ) and GluA3 (shScramble $n=6$ cells; shRab39b $n=7$ cells; Rab39b-rescue $n=9$ cells; Student's $t$-test shScramble versus shRab39b $P=0.03$; Rab39b-rescue versus shRab39b $P=0.04$ ) cell body density in shScramble-, shRab39b- and Rab39b-rescue-treated mouse hippocampal neurons. (b) Quantification of the ratio between mature (1) and immature (2) forms of AMPARs in shRab39b- compared with shScramble-treated neurons after PNGasef or EndoHf digestion. NT: non-treated neurons. Representative western blots (lower panels) showing the maturation ratio for GluA1 ( $n=3$ experimental replicates), GluA2 ( $n=3$ experimental replicates; Student's $t$-test $P=0.002$ ) and GluA3 ( $n=3$ experimental replicates; Student's $t$-test $P=1.28 \mathrm{E}-04)$. (c) Quantification of PICK1 (shScramble $n=14$ cells; shRab39b $n=15$ cells; Rab39b-rescue $n=8$ cells; Student's $t$-test shScramble versus shRab39b $P=0.03$; Rab39b-rescue versus shRab39b $P=0.01$ ), GluA1 (shScramble $n=10$ cells; shRab39b $n=10$ cells; Rab39b-rescue $n=9$ cells; Student's $t$-test shScramble versus shRab39b $P=7.4 \mathrm{E}-04$; Rab39b-rescue versus shRab39b $P=8.1 \mathrm{E}-04$ ), GluA2 (shScramble $n=10$ cells; shRab39b $n=5$ cells; Rab39b-rescue $n=9$ cells; Student's $t$-test shScramble versus

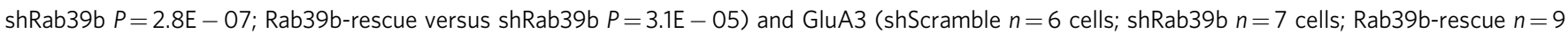
cells; Student's $t$-test shScramble versus shRab39b $P=1.5 \mathrm{E}-05$; Rab39b-rescue versus shRab39b $P=4.7 \mathrm{E}-05$ ) dendrite density in shScramble-, shRab39b- and Rab39b-rescue-treated mouse hippocampal neurons. (d) Representative images of shRab39b, shScramble and Rab39b-rescue neurons immunostained without permeabilization for the extracellular N-terminal region of GluA1 and GluA2. Quantification of positive puncta at cell surface shows a significant increase of GluA1 (shScramble $n=41$ cells; shRab39b $n=40$ cells; Rab39b-rescue $n=10$; Student's $t$-test shScramble versus shRab39b

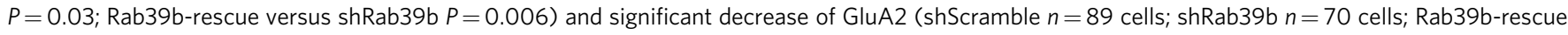
$n=8$; Student's $t$-test shScramble versus shRab39b $P=0.006$; Rab39b-rescue versus shRab39b $P=0.01$ ) subunits. The number of cells belongs from a minimum of three experimental replicates. ${ }^{\star} P<0.05 ;{ }^{\star \star} P<0.01 ;{ }^{\star \star \star} P<0.001$. 

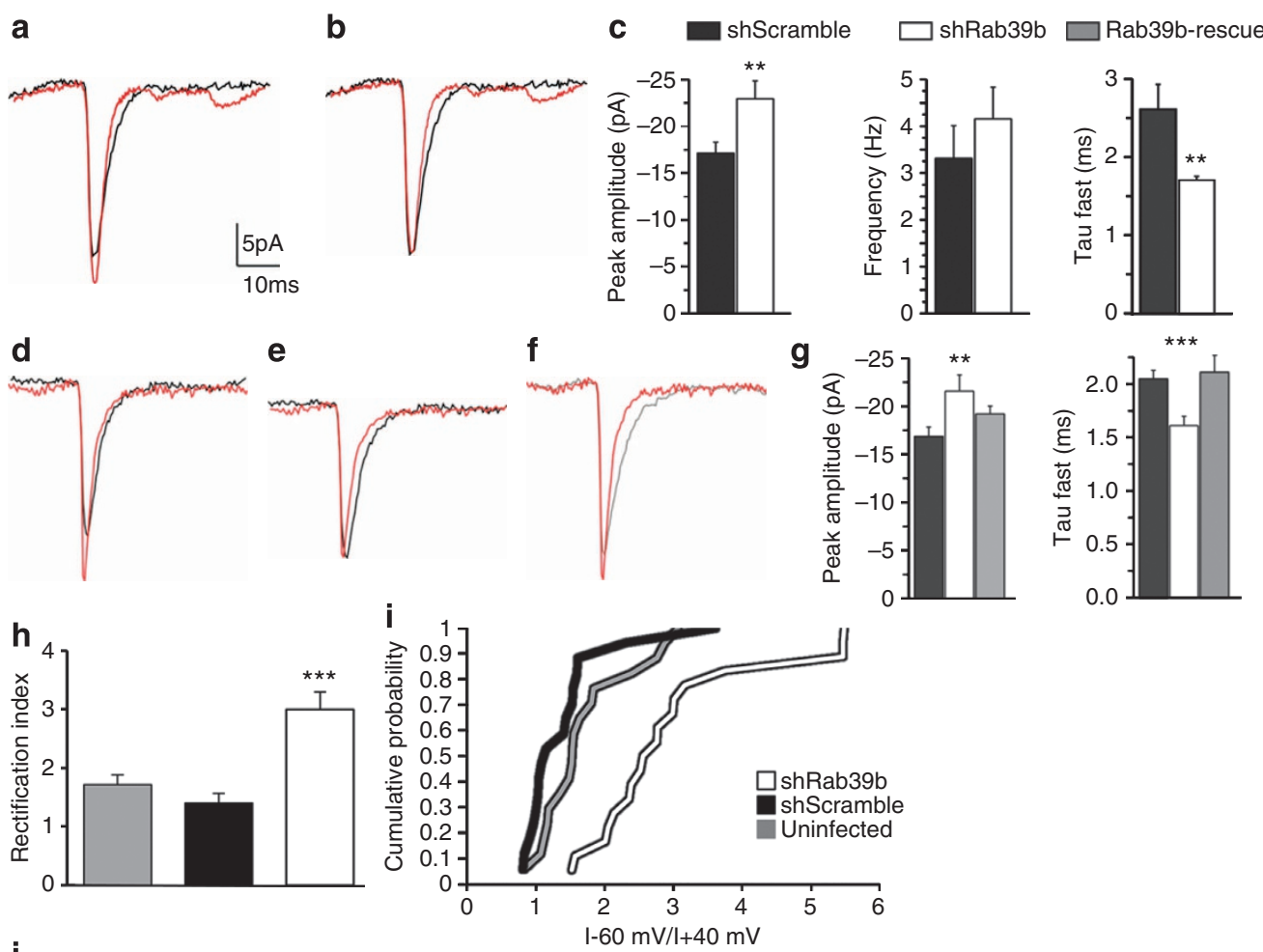

\section{j}

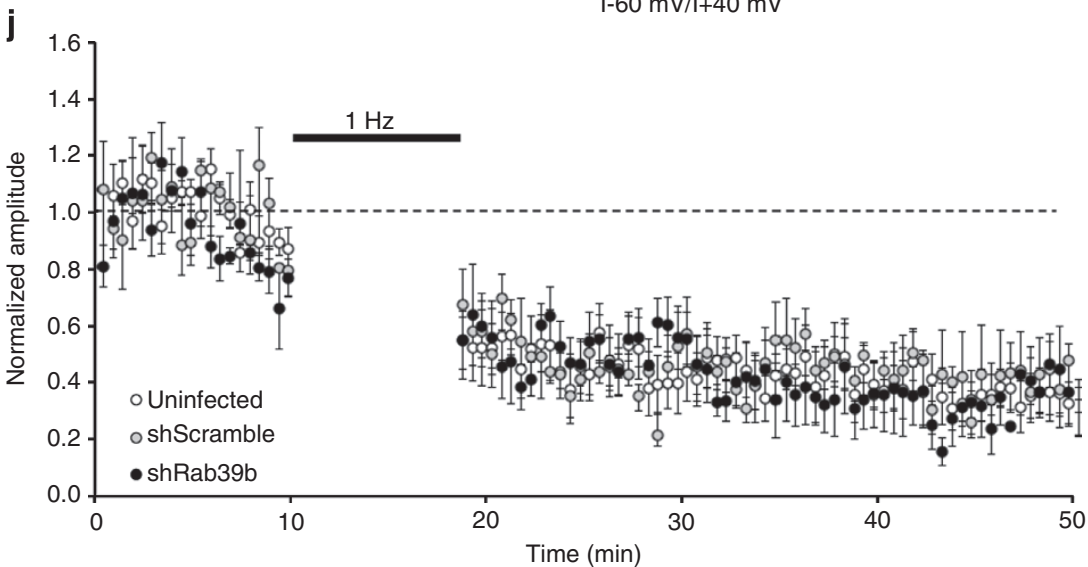

Figure 8 | Synaptic transmission and AMPAR composition. (a) Averages of 100 mEPSCs from shScramble (black trace) and shRab39b (red trace) superimposed showing the peak amplitude increase in silenced neurons (c). In (b) the same traces are superimposed and the silenced average mEPSCs (red) is normalized to the peak of the shScramble (black), revealing the lower decay kinetic of shRab39b currents (c). (c) Histograms show the average changes in the mEPSCs peak amplitude, frequency and decay kinetics (shScramble $n=14$ cells and shRab39b $n=13$ from three experimental replicates; Student's $t$-test $P<0.01$ ). (d) Averages of 100 mEPSCs from shScramble (black) and shRab39b (red) superimposed as in (a) and (e) as in (b). The grey trace in (f) is the average of 100 mEPSCs from Rab39b-rescue neurons, in which the average peak amplitude and kinetics resembles that of the shScramble values (peak amplitude $-19.2 \pm 0.8 \mathrm{pA}$; tau $2.11 \pm 0.21 \mathrm{~ms}, n=10$ cells). These results are summarized in the histograms in ( $\mathbf{g}$ ). The number of cells belongs from a minimum of three experimental replicates. (e) Rectification index histogram from uninfected, shScrambled and shRab39b-treated organotypic slices ( $n=6$ experimental replicates for each condition) and (f) cumulative probability indicate a highly significant increase of the rectification index in Rab39b silenced neurons (Student's $t$-test $P<0,0001$ shRab39b versus shScramble; $P=0,0007$ shRab39b versus uninfected; $P=0,19$ uninfected versus shScramble). (g) LTD representation of uninfected, shScrambled and shRab39b-treated organotypic slices. ${ }^{\star \star} P<0.01 ;{ }^{\star \star \star} P<0.001$.

GluA1/A2 heteromers are mostly confined to post-ER compartments. Finally, to become competent for ER export, AMPARs need to co-assemble with auxiliary proteins. In the brain, auxiliary subunits of the stargazing/TARP (transmembrane AMPAR regulatory protein) family and the cornichon family are physically associated with the channel and regulate their trafficking and gating ${ }^{45-49}$. Recently, Cornichon-2/-3 was described as cargo receptors to selectively bind GluA1, and not GluA2, in hippocampal neurons, allowing the forward trafficking of GluA1-containing AMPARs from the ER to Golgi and finally their synaptic expression ${ }^{50}$. Instead, our study identifies RAB39B as the first RAB GTPase regulating a vesicular trafficking step for the transport of GluA2/GluA3 heteromers into the secretory pathway.

Finally, our analysis of the steady-state levels of AMPAR subunits at the post-synaptic membrane of $R a b 39 b$ silencing hippocampal neurons demonstrates that the alteration in the RAB39B-mediated secretory pathway leads to a decrease in surface GluA2 density and an increase in GluA1 AMPAR subunits. This observation was confirmed functionally by the 


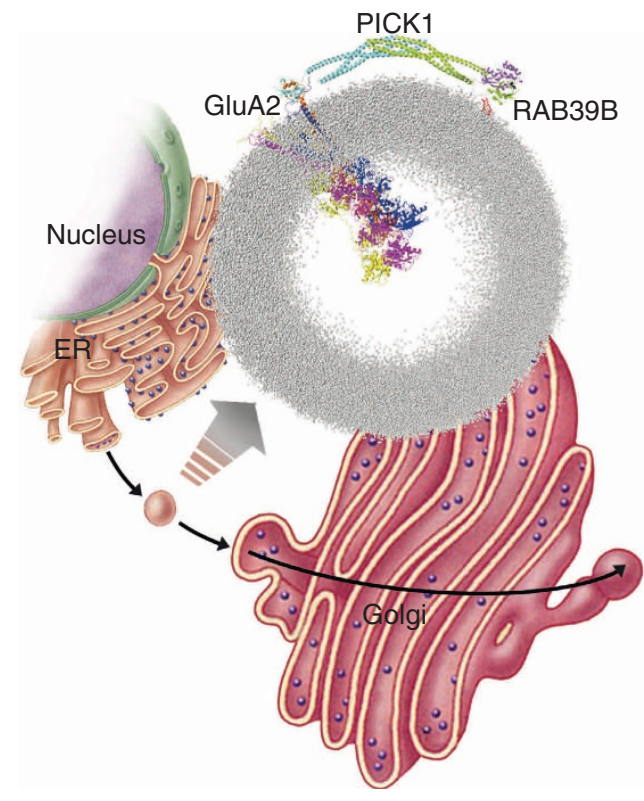

Figure 9 | RAB39B-PICK1-GluA2 model acting at ER-Golgi interface. Proposed model of RAB39B-PICK1-GluA2 complex on a 1,2-dioleoyl-snglycero-3-phosphocholine (DOPC) coarse grained liposome model with a $40 \mathrm{~nm}$ diameter (grey dots are the atoms of DOPC) emphasizing RAB39B-driven traffic of GluA2 cargo between the ER and Golgi compartment.

analysis of the rectification properties of AMPAR-mediated synaptic transmission resulting in an increased rectification index suggestive of an increased fraction of GluA2-lacking AMPARs at synapses related to the increased amplitude of miniature EPSCs. Because it was previously described that, in the hippocampus, heterotetramers of GluA1/GluA2 and GluA2/GluA3 subunits, together with a smaller contribution from GluA1 homomers, represent the most common combinations in excitatory synapses $^{22}$, we could speculate that altering RAB39B-mediated early trafficking step of GluA2/GluA3, the result will be an increase in GluA1 $\mathrm{Ca}^{2+}$-permeable homomers.

In fact, the AMPA-type glutamate receptors play a critical role in synaptic plasticity underlying learning and memory by mediating the majority of fast excitatory synaptic transmission and by trafficking into and out of the synapse $\mathrm{e}^{51}$. The presence of GluA2 in heteromeric AMPAR renders the channel impermeable to $\mathrm{Ca}^{2+}$ and $\mathrm{Zn}^{2+}$, thus influencing channel kinetic, channel conductance and synaptic transmission. In contrast, GluA2lacking AMPAR is $\mathrm{Ca}^{2+}$-permeable and is mostly observed in young animals or after an epoch of plasticity-inducing neuronal activity ${ }^{52-54}$. In fact, our results describe a slight alteration in spine morphology, depicting a scenario of immature spines in shRab39b-treated neurons, as they are shorter and wider than control neurons. Previous studies described that $\mathrm{Ca}^{2+}$-permeable AMPARs are important in experience-dependent and synaptic plasticity ${ }^{55}$. These studies suggest that $\mathrm{Ca}^{2+}$-permeable AMPAR play a prominent role in maintaining circuits in a labile state where further plasticity can occur, thus promoting metaplasticity ${ }^{56}$. Moreover, it was shown that the abnormal expression of $\mathrm{Ca}^{2+}$-permeable AMPARs is implicated in drug addiction and memory disorders, with significant implication in the development of therapeutic approaches to these disorders ${ }^{56}$. In addition, alteration of the relative proportion of AMPAR subunits after pathological insults ${ }^{54,57}$ or in response to physiological stimuli ${ }^{58}$ will shift the balance towards homomers and in turn alter the signalling landscape of the neuron.
Altogether our findings shed light on the molecular mechanisms responsible for the maturation and the trafficking of GluA2/ GluA3 AMPAR in the secretory pathway, and pave the way to explain the functional role of $\mathrm{RAB} 39 \mathrm{~B}$ in cognitive disorder.

Unsolved questions remain still open. At first, what other molecules convey the transport competence to RAB39B? One possibility could be the involvement of additional downstream effectors proteins that give the directionality and the timing for RAB39B-PICK1-GluA2 complex movement. GM130 a cis-Golgi protein involved in accepting vesicles from the $\mathrm{ER}^{59,60}$, as well as dynein/dynactin motor complex, driving microtubule-dependent trafficking from the ER to Golgi ${ }^{61}$ and along dendrites to the post-synaptic sites ${ }^{62}$ or myosin $\mathrm{Va}^{63}$ involved in dendritic and post-synaptic actin-dependent trafficking ${ }^{64}$ could be the most reliable factors. At present, RAB39B is required for the transport of GluA2-containing AMPAR from the ER to the Golgi, but we cannot assess its involvement from the Golgi to the neuronal surface. Future studies will undoubtedly be necessary to answer these questions as well as the availability of Rab39b-null mouse model to better define the link between RAB39B-mediated intracellular trafficking and cognitive disorders.

\section{Methods}

Most of the experiments were on mouse hippocampal neurons prepared from mouse embryos at E18 days. Astroglial feeder layer cultures were prepared from mouse cortices at post-natal day 2. Mice were $\mathrm{C} 57 \mathrm{Bl} / 6 \mathrm{~N}$ genetic background and were obtained from Charles River, Italy. Mice were euthanized in accordance with 'Institutional Animal Care and Use Committee San Raffaele (IACUC)' at San Raffaele Scientific Institute, Milan, Italy and approved by the Italian National Ministry of Health, IACUC ID 470 and in accordance with the guidelines established by the European Community Council Directive of 24 November 1986 on the use of animals in research (86/609/EEC).

Plasmids generation. pGEX-RAB5 and pGEM-p110 $\beta$ were a gift from Professor M. Zerial (Max Planck, Dresden, Germany). GST-RAB39A was a gift from Professor M. Fukuda (Tohoku Univ., Japan). Myc-PICK1, pGEX-PICK1, myc-PDZ PICK1, flag- $\Delta 121$ PICK1, myc-KDAA PICK1, myc-K83A PICK1, GFP-GluA1 and GFP-GluA2 were a gift from Dr M. Passafaro (CNR Institute of Neuroscience, Milan, Italy) and Professor R. Huganir (Johns Hopkins University, Baltimore, MD, USA). pGEM-PICK1 was created amplifying the full-length rat Pick1 coding sequence using specific primers (PICK1 For: 5'-GCGCCACCATGTTTGCAGACTTAGAC

TATGACAT-3'; PICK1 Rev: 5' -TCAGGAGTCACACCAGCTTCCG-3'). In the forward primer the KOZAK sequence (CCACC) was inserted. The PCR product was then cloned into pGEM-T-easy (Promega). Flag-RAB39B was created as previously described ${ }^{10}$. pGEX-RAB39B was created amplifying the mouse Rab39b cDNA from flag-RAB39B vector using specific primers (pPC86 For: $5^{\prime}$-GCGGT CGACCATGGAGGCCATCTGGCTGTACC-3'; pGEX Rev: 5'-GCGGCGGCCGC CTAGCACAAACATCTCCTCTCTGA-3'), and the PCR product was than cloned in frame into SalI and NotI sites of p-GEX-4T-2 plasmid (GE Healthcare Life Sciences).

The site-directed mutagenesis using the Quick Change Lightning kit (Stratagene) was used to generate nucleotide deletion or mutations on flag-RAB39B or pGEX-RAB39B to obtain the following plasmids: flag- $\triangle \mathrm{ER}$ (deletion of 45 nucleotides from +105 to +147 ; deletion of 15 amino acids from +35 to +49 ), flag-D38A (mutations on nucleotides from +112 to +114 , mutation on amino acid +38 , D38A), flag-V41G42V43AAA (mutations on nucleotides from +121 to +129 , mutations on amino acids from +41 to +43 , V41A, G42A, V43A), flagD44F45AA (mutations on nucleotides from +130 to +135 , mutations on amino acids from +44 to +45 , D44A, F45A), pGEX-RAB39B S22N (mutations on nucleotides from +63 to +65 , mutation on amino acid +22 , S22N), pGEXRAB39B Q68L (mutations on nucleotides from +64 to +66 , mutation on amino acid +22 , Q68L). pDBleu-RAB39B was created amplifying the human $R A B 39 B$ cDNA, obtained from retro-transcription of linfoblastoid cells RNA from control patients, using specific primers (pPC86 For: 5'-GCGGTCGACCATGGAGGCC ATCTGGCTGTACC-3'; pPC86 Rev: $5^{\prime}$-GCGGCGGCCGCTCTAGCACAAACA TCTCCTCTCTGA-3' ${ }^{\prime}$. The PCR product was than cloned in frame into SalI and NotI sites of pDB-Leu (ProQuest Two-hybrid system, Gibco BRL). FLAGRAB39B-rescue, cherryRAB39B-rescue and GFP-RAB39A was created as previously described ${ }^{10}$.

Yeast two-hybrid assay. Yeast two hybrid screening was performed following the manufacturer's instruction (ProQuest Two-hybrid system, Gibco BRL). The fulllength human $R A B 39 B$ cDNA was cloned into the pDBLeu vector, in frame with 
the GAL4-binding domain, and used as bait to screen a human foetal brain cDNA library (ProQuest Pre-made cDNA Libraries) cloned into the pPC86 vector in frame with the GAL4-activating domain. Positive colonies grew on plates containing $10 \mathrm{mM} \mathrm{3-amino-1,2,4-triazole(3-AT)} \mathrm{without} \mathrm{tryptophan} \mathrm{and} \mathrm{leucine} \mathrm{and}$ expressed different reporter genes: HIS3 and URA3 allowed the growth, respectively, in medium lacking histidine and uracil, LacZ induced a colorimetric reaction in the presence of 5-bromo-4-chloro-3-indolyl- $\beta$-D-galactopyranoside (X-gal). cDNA plasmids from positive clones were recovered via TOP10F' E. coli, plated on ampicillin and sequenced. Yeast two-hybrid screening was repeated two times and only positive colonies expressing three reporter genes (HIS3, LacZ and URA3) were taken in consideration. The test was performed following the manufacturer's instructions (ProQuest Two-hybrid system, Gibco BRL).

GST pull-down. GST fusion proteins were prepared in E. coli strain BL21DE3, isolated and immobilized on glutathione-Sepharose 4B beads (GE Healthcare; as decribed ${ }^{65}$ ). GST pull-down experiments were carried out with mouse brain lysates or COS7 cells transfected with different constructs or in vitro transcribed and translated PICK1.

A mouse brain was lysed with HKT buffer ( $4 \mathrm{mM}$ Hepes pH 7.4, $400 \mathrm{mM} \mathrm{KCl}$, $4 \mathrm{mM}$ EDTA, $2 \%$ Triton X-100, Protease Inhibitor cocktail) and rotated at $4{ }^{\circ} \mathrm{C}$ for $45 \mathrm{~min}$ before dilution 1:2 with HKT buffer and centrifuging at $16,000 \mathrm{~g}$ for $10 \mathrm{~min}$ to pellet debris, or it was lysed with RIPA buffer ( $500 \mathrm{mM}$ TrisHCl pH 7.4, $200 \mathrm{mM}$ $\mathrm{NaCl}, 1 \mathrm{mM}$ EDTA, 1\% Triton X-100, 1\% NP-40, Protease Inhibitor cocktail) and rotated at $4{ }^{\circ} \mathrm{C}$ for $1 \mathrm{~h}$ before centrifuging at $500 \mathrm{~g}$ for $10 \mathrm{~min}$ to pellet debris.

COS7 cells were transfected with plasmids expressing different PICK1 constructs as myc-PDZ, flag- $\Delta 121$, myc-KDAA, myc-K83A, or different RAB39B constructs as flag-RAB39B, flag- $\triangle E R$, flag-D38A, flag-V41G42V43AAA, flagD44F45AA. After 2 days of transfection, COS7 cells were lysed in RIPA buffer, rotated at $4{ }^{\circ} \mathrm{C}$ for $1 \mathrm{~h}$ before centrifuging at $12,500 \mathrm{~g}$ for $20 \mathrm{~min}$ to pellet debris and sonicated for $5 \mathrm{~min}$.

PICK1 was in vitro transcribed and translated modifying the standard protocol (GeHealthcare) as follows: the in vitro reaction was reduced to $30 \mathrm{~min}$ instead of $90 \mathrm{~min}$.

All the lysates were pre-cleared with $120 \mu \mathrm{g}$ GST immobilized on glutathioneSepharose $4 \mathrm{~B}$ beads (GE Healthcare) for $1 \mathrm{~h}$ at $4{ }^{\circ} \mathrm{C}$.

Forty micrograms of GST-RAB39B in presence of GDP or GTP $\gamma \mathrm{S}$ (SigmaAldrich) or $40 \mu \mathrm{g}$ of GST-RAB39B S22N and GST-RAB39B Q68L were incubated on rotation $3 \mathrm{~h}$ at $4{ }^{\circ} \mathrm{C}$ with $8 \mathrm{mg}$ of mouse brain lysed in HKT or RIPA buffer, or $2 \mathrm{mg}$ of COS7 cells expressing myc-PDZ, flag- $\Delta 121$, myc-KDAA, myc-K83A or $10 \mu \mathrm{l}$ of the in vitro translational reaction of PICK1. Eighty micrograms of GSTPICK1 immobilized on glutathione-Sepharose 4B beads were incubated on rotation $3 \mathrm{~h}$ at $4{ }^{\circ} \mathrm{C}$ with $16 \mathrm{mg}$ of mouse brain lysed in HKT buffer or $2 \mathrm{mg}$ of COS7 cells expressing flag-RAB39B or flag-RAB39B $\triangle \mathrm{ER}$, flag-D38A, flag-V41G42V43AAA or flag-D44F45AA.

In all the experiments, GST alone and/or GST-RAB5 GDP and GTP $\gamma$ S immobilized on glutathione-Sepharose $4 \mathrm{~B}$ beads were used as control.

After washing, the proteins are eluted with elution buffer $(25 \mathrm{mM}$ Reduced Glutathione, $500 \mathrm{mM}$ Tris $\mathrm{pH} 8,300 \mathrm{mM} \mathrm{NaCl}$, Protease Inhibitor cocktail) for 30 min at RT, resuspended in $5 \times$ SDS sample buffer $(4 \%$ SDS, $1.3 \mathrm{M}$ Sucrose, $5 \mathrm{mM}$ EDTA, $10 \% \beta$-mercaptoethanol, $0.5 \mathrm{M}$ Tris $\mathrm{pH} 6.8$ ), analysed by SDS-PAGE and western blot with appropriate antibodies: the anti-PICK1 polyclonal antibody (Abcam, \#ab3420, 1:500 WB), the anti-PKC $\alpha$ polyclonal antibody (Cell Signaling, \#2056, 1:1,000 WB), anti-p110 $\beta$ polyclonal antibody (Santa Cruz, \#sc-602, 1:200 $\mathrm{WB}$ ), the anti-RAB39B polyclonal antibody (produced by BioGenes against the hypervariable C-terminal RAB39B sequence CVVHSSEEVIKSERR and tested for binding specificity as showed in Supplementary Fig. 1d; 1:500 WB), anti-myc monoclonal antibody (Sigma-Aldrich; \#M4439; 1:5,000 WB) and the anti-FLAG polyclonal antibody (Sigma-Aldrich, \#F1804, 1:1,000 WB), anti-GluA2 monoclonal antibody (Millipore, \#MAB397, 1:200 IF), anti-RAB5 monoclonal antibody (BD Transduction, \#610282, 1:500 WB) and anti-GST polyclonal antibody (Santa Cruz, \#sc-459, 1:500 WB). Band intensity was measured with ImageJ.

Immunoprecipitation. Immunoprecipitation studies were carried out with lysates prepared from HEK293T cells co-expressing flag-RAB39B and myc-PICK1 or from wild-type mouse brain or Pick1-knockout mouse brain. Transfected cells were washed in phosphate-buffered saline (PBS) $\mathrm{Ca}^{2+} / \mathrm{Mg}^{2+}(0.1 \mathrm{mM} \mathrm{CaCl}, 1 \mathrm{mM}$ $\mathrm{MgCl}_{2}$ ) and scraped in CHAPS buffer (1\% CHAPS; Sigma-Aldrich; $0.1 \mathrm{mM}$ EDTA $\mathrm{pH} 8$, Protease Inhibitor Cocktail in PBS solution) and rotated at $4{ }^{\circ} \mathrm{C}$ for $1 \mathrm{~h}$ before centrifuging at $13,200 \mathrm{~g}$ for $20 \mathrm{~min}$ to pellet debris. Mouse brains were lysed in HBST buffer (10 mM HEPES pH 7.4, $150 \mathrm{mM} \mathrm{NaCl}, 0.5 \%$ Triton X-100, Protease Inhibitor Cocktail in $\mathrm{H}_{2} \mathrm{O}$ solution), homogenized 10 times by hands, rotated at $4{ }^{\circ} \mathrm{C}$ for $10 \mathrm{~min}$ and centrifuged at $13,500 \mathrm{~g}$ for $20 \mathrm{~min}$ at $+4^{\circ} \mathrm{C}$ to pellet debris

All the lysates were pre-cleared with washed protein G-Sepharose-4 fast flow beads (GE Healthcare/Amersham Biosciences) for $2 \mathrm{~h}$ at $4{ }^{\circ} \mathrm{C}$.

Immunoprecipitation studies were performed, in presence of $3 \mathrm{mM} \mathrm{GTP} \gamma \mathrm{S}$ (Sigma-Aldrich), incubating HEK293T cells transfected with flag-RAB39B and myc-PICK1 with an anti-myc monoclonal antibody ( $6 \mu$ g; Sigma-Aldrich; \#M4439) for $3 \mathrm{~h}$ at $4{ }^{\circ} \mathrm{C}$ on rotation, and wild type or Pick 1 KO mouse brain lysates with an anti-GluA2 monoclonal antibody ( $5 \mu \mathrm{g}$; Millipore; \#MAB397) $24 \mathrm{~h}$ at $4{ }^{\circ} \mathrm{C}$ on rotation. IgG $(5 \mu \mathrm{g})$ was used as a control.
The beads were eluted in $5 \times$ SDS sample buffer ( $4 \%$ SDS, $1.3 \mathrm{M}$ Sucrose, $5 \mathrm{mM}$ EDTA, $10 \% \beta$-mercaptoethanol, $0.5 \mathrm{M}$ Tris $\mathrm{pH} 6.8) 10 \mathrm{~min}$ at room temperature and analysed by $4-12 \%$ polyacrylamide gel on SDS-PAGE and western blot with appropriate antibodies: anti-myc monoclonal antibody (Covance, \#MMS-150P, 1:5,000 WB), anti-FLAG polyclonal antibody (Sigma-Aldrich, \#F7425, 1:1,000 WB), anti-GluA2 polyclonal antibody (SySy, \#182103, 1:1,000 WB), anti-PICK1 polyclonal antibody (JH2906 was made into the laboratory of Prof. R. Huganir Johns Hopkins University, Baltimore, MD, USA, 1:250 WB), anti-RAB39B polyclonal antibody (produced by BioGenes against the hypervariable C-terminal RAB39B sequence CVVHSSEEVIKSERR; 1:500 WB).

Cell cultures, transfection and lentiviral transduction. COS7 and HEK293T cells were grown in Dulbecco's modified Eagle's medium (Gibco) supplemented with $10 \%$ fetal bovine serum (Gibco), $1 \%$ glutamine, $1 \%$ penicillin and streptomycin and were regularly passaged to maintain exponential growth. COS7 cells were transfected using Lipofectamine 2000 (Invitrogen) following the manufacturer's instructions and fixed $24 \mathrm{~h}$ after transfection.

Mouse hippocampal neurons were transduced (MOI1) at DIV1 with lentiviral particles expressing shScramble or shRab39b as previously described ${ }^{10}$. See Supplementary Experimental Procedures for details.

Deglycosilation. Instructions for the use of EndoHf and PNGasef (NEB) were followed according to the manufacturer's instructions to digest proteins, with minor modifications. In brief, cultured hippocampal neurons transduced with shRab39b or shScramble were lysed with Lysis Buffer (1\% SDS, 2 mM EDTA, $10 \mathrm{mM}$ hepes pH 7,4, Protease Inhibitor Cocktail). A total of $260 \mu \mathrm{g}$ of lysed were for denatured $15 \mathrm{~min}$ at $55^{\circ} \mathrm{C}$ and digested with EndoHf or PNGasef for $2 \mathrm{~h}$ at $37^{\circ} \mathrm{C}$. SDS loading buffer $(5 \times)$ was finally added to samples, which were split in three different parts, 30, 30 and $200 \mu \mathrm{g}$, and analysed by western blot with $6 \%$ SDSPAGE. GluA1, GluA2 and GluA3 were detected using a anti-GluA1 polyclonal antibody (SySy, \#182003, 1:1,000 WB), anti-GluA2 polyclonal antibody (SySy; \#182103, 1:1,000 WB) and anti-GluA3 polyclonal antibody (made into the laboratory of Professor R. Huganir Johns Hopkins University, Baltimore, MD, USA, 1:1,000 WB).

Immunofluorescence. Standard immunofluorescence experiments were carried out by fixing the cells for $15 \mathrm{~min}$ with $4 \%$ paraformaldehyde, $4 \%$ sucrose (SigmaAldrich) in $120 \mathrm{mM}$ sodium phosphate buffer, $\mathrm{pH}$ 7.4. Coverslips were rinsed three times with PBS and then incubated overnight at $4{ }^{\circ} \mathrm{C}$ into a humidified chamber with the primary antibody (anti-flag polyclonal antibody: Sigma-Aldrich; poly \#F7425, 1:200 IF, anti-flag monoclonal antibody: Sigma-Aldrich, \#F1804, 1:400 IF; anti-myc monoclonal antibody: Sigma-Aldrich; \#M4439; 1:5,000 WB and 1:400 IF; anti-PICK1: Thermo Scientific, \#PA1-073, 1:100 IF; anti-calreticulin: Sigma, \#C4606, 1:500 IF; anti-GM130: BD Transduction, \#610823, 1:50 IF) appropriately diluted in goat serum dilution buffer (GSDB; $15 \%$ goat serum, $450 \mathrm{mM} \mathrm{NaCl}, 0.3 \%$ Triton X-100, $20 \mathrm{mM}$ sodium phosphate buffer, $\mathrm{pH}$ 7.4). Coverslips were washed three times within $30 \mathrm{~min}$ with high salt buffer (HS: $500 \mathrm{mM} \mathrm{NaCl}, 20 \mathrm{mM}$ sodium phosphate buffer, $\mathrm{pH}$ 7.4) and then incubated with the appropriate secondary antibodies (Molecular Probes, Invitrogen) for $90 \mathrm{~min}$ at room temperature. After three washes with HS over $30 \mathrm{~min}$ and one wash with $5 \mathrm{mM}$ sodium phosphate buffer, $\mathrm{pH}$ 7.4, and coverslips were mounted with Vectashield (Vectalab).

Visualization of intracellular endogenous AMPAR subunits in neurons was done by hiding the portion of receptors exposed on the membrane surface with primary monoclonal antibodies recognizing the $\mathrm{N}$-terminal portion (anti-GluAl: Millipore, \#MAB2263, 1: 1:200 IF; anti-GluA2: Millipore, \#MAB397, 1:200 IF; antiGluA3: made into the laboratory of Prof. R. Huganir Johns Hopkins University, Baltimore, MD, USA, 1:100 IF) diluted in GSDB without Triton X-100 as well as the HRP-conjugated secondary antibody (Biorad, 1:200), and then cells were incubated with the primary monoclonal antibodies recognizing the N-terminal portion diluted in GSDB with $0.3 \%$ Triton X-100 as well as the appropriate secondary antibody (Molecular Probes, Invitrogen).

To visualize GluA1 and GluA2 exposed on the cell surface, the primary monoclonal antibodies recognizing the N-terminal portion were diluted in GSDB without Triton X-100, as well as the secondary antibody (Molecular Probes, Invitrogen).

Image acquisition and analysis. For Western blots, ImageJ Analysis Software ('Analyze gels' plugin) was used; full scan of western blots is shown in Supplementary Fig. 6. For GST pull-down experiments, PICK1 binding to GSTRAB39B GDP versus GST-RAB39B GTP $\gamma$ S is quantified as follow: signal from GST-RAB39B GTP $\gamma$ S binding protein is normalized on the $50 \mathrm{kDa}$ GST signal and set at 100\%; consequently, the level of GST-RAB39B GDP binding protein, normalized on the $50 \mathrm{KDa}$ GST band, is expressed as percentage of 100 .

Confocal images of COS7 cells were obtained using Leica SP8 SMD laser scanning confocal. For spine morphology fluorescent images were acquired with a Biorad MRC1024 confocal microscope, using a Nikon $60 \times$ objective with sequential acquisition setting at 1,280 $\times 1,024$ pixel resolution and image data were a $z$ series projection of about $5-10$ images, each averaged 4 times and taken at $0.7 \mu \mathrm{m}$ depth intervals. 
Pictures of immunostained hippocampal neurons were captured with the same exposure conditions using DeltaVision microscope (Applied Precision) equipped with a $\times 60$ or $\times 100$ objectives. At least $10 \mathrm{Z}$-space slices of $0.20 \mu \mathrm{m}$ were deconvolved and flattened by maximum projection.

TIRF images, setting $110 \mathrm{~nm}$ as the distance from the coverslip, were acquired using Leica SR GSD 3D TIRF microscope (Leica).

Pearson's correlation coefficient was calculated using Volocity software to measure the correlation between two proteins.

Morphometric spine measurements were made with NeuronStudio software.

ImageJ Analysis Software ('Gran filter' plugin setting the size from 1 to infinity) was used to measure AMPAR subunits and PICK1 density relatively to the area of infected cell body and dendrites.

Electrophysiology in cultured hippocampal neurons. Seventeen to twenty DIV hippocampal neurons transduced at low or high MOI with shScramble or shRab39b, and in some case transfected with Rab39b as rescue, were patched and whole-cell recordings were made on infected neurons held at $-70 \mathrm{mV}$ in the presence of $3 \mu \mathrm{M}$ TTX. mEPSCs were acquired until the cell was stable and analysed using pClamp10 (Molecular Devices). Threshold mEPSC amplitude was set at $5 \mathrm{pA}$, and $300-500$ events were collected and averaged to calculate the mean mEPSC amplitude, frequency and kinetics for each culture preparation examined. See Supplementary Experimental Procedures for details.

Electrophysiology in organotypic hippocampal slices. Hippocampal slice cultures were prepared from postnatal day 5-7 mice C57BL/6N. After 1 day in culture every slice was transduced with lentiviral particles (shScramble or shRab39b). After 10-12 days in culture, simultaneous voltage-clamp whole-cell recordings were obtained from nearby infected and uninfected CA1 pyramidal neurons. Whole-cell recordings were made with a Multiclamp 700B amplifier (Axon Instruments) and electrophysiological data were collected with pCLAMP software (Molecular Devices). See Supplementary Experimental Procedures for details.

Statistical analysis. Data are expressed as mean \pm s.e.m. Statistical significance was assessed using paired Student's $t$-test as appropriate (for two groups comparison). We verified the difference between groups by means of $\mathrm{t}$-type test statistic and exact $P$ values computed with permutation methods to avoid any distributional assumption or asymptotical approximation. $P$ values were, then, adjusted for multiplicity applying the Benjamini-Hochberg procedure (false discovery rate $)^{66}$.

Molecular modelling. The structural models of PICK1 and RAB39B were achieved by comparative modelling (that is, by MODELLER ${ }^{67}$ ), whereas the interaction between them was predicted by protein docking (that is, by ZDOCK ${ }^{68}$ ). The CTD of GluA2 was built by comparative modelling as well. Details of Computational modelling are provided as Supplementary Information.

\section{References}

1. Stenmark, H. \& Olkkonen, V. M. The Rab GTPase family. Genome. Biol. 2, REVIEWS3007 (2001)

2. Zerial, M. \& McBride, H. Rab proteins as membrane organizers. Nat. Rev. 2, 107-117 (2001).

3. Barr, F. A. Review series: Rab GTPases and membrane identity: causal or inconsequential? J. Cell. Biol. 202, 191-199 (2013).

4. Corbeel, L. \& Freson, K. Rab proteins and Rab-associated proteins: major actors in the mechanism of protein-trafficking disorders. Eur. J. Pediatr. 167, 723-729 (2008).

5. D'Adamo, P. et al. RAB GTPases and RAB-interacting proteins and their role in the control of cognitive functions. Neurosci. Biobehav. Rev. 46 Pt 2, 302-314 (2014).

6. D'Adamo, P. et al. Mutations in GDI1 are responsible for X-linked non-specific mental retardation. Nat. Genet. 19, 134-139 (1998).

7. Strobl-Wildemann, G. et al. Novel GDI1 mutation in a large family with nonsyndromic X-linked intellectual disability. Am. J. Med. Genet. A. 155A, 3067-3070 (2011).

8. Bianchi, V. et al. Cognitive impairment in Gdil-deficient mice is associated with altered synaptic vesicle pools and short-term synaptic plasticity, and can be corrected by appropriate learning training. Hum. Mol. Genet. 18, 105-117 (2009).

9. Bianchi, V. et al. Forebrain deletion of alphaGDI in adult mice worsens the presynaptic deficit at cortico-lateral amygdala synaptic connections. PLoS ONE 7, e29763 (2012)

10. Giannandrea, M. et al. Mutations in the small GTPase gene RAB39B are responsible for X-linked mental retardation associated with autism, epilepsy, and macrocephaly. Am. J. Hum. Genet. 86, 185-195 (2010).

11. Wilson, G. R. et al. Mutations in RAB39B cause X-linked intellectual disability and early-onset Parkinson disease with alpha-Synuclein Pathology. Am. J. Hum. Genet. 95, 729-735 (2014).
12. Vanmarsenille, L. et al. Increased dosage of RAB39B affects neuronal development and could explain the cognitive impairment in male patients with distal Xq28 copy number gains. Hum. Mutat. 35, 377-383 (2013).

13. Perez, J. L. et al. PICK1 targets activated protein kinase Calpha to AMPA receptor clusters in spines of hippocampal neurons and reduces surface levels of the AMPA-type glutamate receptor subunit 2. J. Neurosci. 21, 5417-5428 (2001).

14. Esteban, J. A. AMPA receptor trafficking: a road map for synaptic plasticity. Mol. Interv. 3, 375-385 (2003).

15. Hanley, J. G. \& Henley, J. M. PICK1 is a calcium-sensor for NMDA-induced AMPA receptor trafficking. EMBO J. 24, 3266-3278 (2005).

16. Hanley, J. G. Molecular mechanisms for regulation of AMPAR trafficking by PICK1. Biochem. Soc. Trans. 34, 931-935 (2006).

17. Anggono, V., Clem, R. L. \& Huganir, R. L. PICK1 loss of function occludes homeostatic synaptic scaling. J. Neurosci. 31, 2188-2196 (2011).

18. Anggono, V. \& Huganir, R. L. Regulation of AMPA receptor trafficking and synaptic plasticity. Curr. Opin. Neurobiol. 22, 461-469 (2012).

19. Traynelis, S. F. et al. Glutamate receptor ion channels: structure, regulation, and function. Pharmacol. Rev. 62, 405-496 (2010).

20. Rossmann, M. et al. Subunit-selective N-terminal domain associations organize the formation of AMPA receptor heteromers. EMBO J. 30, 959-971 (2011).

21. Salussolia, C. L. et al. Interaction of the M4 segment with other transmembrane segments is required for surface expression of mammalian alpha-amino-3hydroxy-5-methyl-4-isoxazolepropionic acid (AMPA) receptors. J. Biol. Chem. 286, 40205-40218 (2011).

22. Wenthold, R. J., Petralia, R. S., Blahos, II J. \& Niedzielski, A. S. Evidence for multiple AMPA receptor complexes in hippocampal CA1/CA2 neurons. J. Neurosci. 16, 1982-1989 (1996).

23. Leonard, A. S., Davare, M. A., Horne, M. C., Garner, C. C. \& Hell, J. W. SAP97 is associated with the alpha-amino-3-hydroxy-5-methylisoxazole-4-propionic acid receptor GluR1 subunit. J. Biol. Chem. 273, 19518-19524 (1998).

24. Sans, N. et al. Synapse-associated protein 97 selectively associates with a subset of AMPA receptors early in their biosynthetic pathway. J. Neurosci. 21, 7506-7516 (2001).

25. Greger, I. H., Khatri, L. \& Ziff, E. B. RNA editing at arg607 controls AMPA receptor exit from the endoplasmic reticulum. Neuron 34, 759-772 (2002).

26. Cull-Candy, S., Kelly, L. \& Farrant, M. Regulation of Ca2 +-permeable AMPA receptors: synaptic plasticity and beyond. Curr. Opin. Neurobiol. 16, 288-297 (2006).

27. Staudinger, J., Zhou, J., Burgess, R., Elledge, S. J. \& Olson, E. N. PICK1: a perinuclear binding protein and substrate for protein kinase $\mathrm{C}$ isolated by the yeast two-hybrid system. J. Cell. Biol. 128, 263-271 (1995).

28. Staudinger, J., Lu, J. \& Olson, E. N. Specific interaction of the PDZ domain protein PICK1 with the $\mathrm{COOH}$ terminus of protein kinase C-alpha. J. Biol. Chem. 272, 32019-32024 (1997).

29. Xia, J., Zhang, X., Staudinger, J. \& Huganir, R. L. Clustering of AMPA receptors by the synaptic PDZ domain-containing protein PICK1. Neuron 22, 179-187 (1999).

30. Pfeffer, S. R. Structural clues to Rab GTPase functional diversity. J. Biol. Chem. 280, 15485-15488 (2005)

31. Madsen, K. L. et al. Molecular determinants for the complex binding specificity of the PDZ domain in PICK1. J. Biol. Chem. 280, 20539-20548 (2005).

32. Xu, J. \& Xia, J. Structure and function of PICK1. Neurosignals 15, 190-201 (2006).

33. Dev, K. K., Nakanishi, S. \& Henley, J. M. The PDZ domain of PICK1 differentially accepts protein kinase C-alpha and GluR2 as interacting ligands. J. Biol. Chem. 279, 41393-41397 (2004).

34. Thorsen, T. S. et al. Identification of a small-molecule inhibitor of the PICK1 PDZ domain that inhibits hippocampal LTP and LTD. Proc. Natl Acad. Sci. USA 107, 413-418 (2010).

35. Greger, I. H., Ziff, E. B. \& Penn, A. C. Molecular determinants of AMPA receptor subunit assembly. Trends Neurosci. 30, 407-416 (2007).

36. Jin, W. et al. Lipid binding regulates synaptic targeting of PICK1, AMPA receptor trafficking, and synaptic plasticity. J. Neurosci. 26, 2380-2390 (2006).

37. Pan, L. et al. Clustering and synaptic targeting of PICK1 requires direct interaction between the PDZ domain and lipid membranes. EMBO J. 26, 4576-4587 (2007).

38. Bowie, D. \& Mayer, M. L. Inward rectification of both AMPA and kainate subtype glutamate receptors generated by polyamine-mediated ion channel block. Neuron 15, 453-462 (1995).

39. Swanson, G. T., Kamboj, S. K. \& Cull-Candy, S. G. Single-channel properties of recombinant AMPA receptors depend on RNA editing, splice variation, and subunit composition. J. Neurosci. 17, 58-69 (1997).

40. Kim, C. H., Chung, H. J., Lee, H. K. \& Huganir, R. L. Interaction of the AMPA receptor subunit GluR2/3 with PDZ domains regulates hippocampal long-term depression. Proc. Natl Acad. Sci. USA 98, 11725-11730 (2001).

41. Makuch, L. et al. Regulation of AMPA receptor function by the human memory-associated gene KIBRA. Neuron 71, 1022-1029 (2011). 
42. Bassani, S. et al. The X-linked intellectual disability protein TSPAN7 regulates excitatory synapse development and AMPAR trafficking. Neuron 73, $1143-1158$ (2012).

43. Hurtley, S. M. \& Helenius, A. Protein oligomerization in the endoplasmic reticulum. Annu. Rev. Cell Biol. 5, 277-307 (1989).

44. Ellgaard, L., Molinari, M. \& Helenius, A. Setting the standards: quality control in the secretory pathway. Science 286, 1882-1888 (1999).

45. Chen, L. et al. Stargazin regulates synaptic targeting of AMPA receptors by two distinct mechanisms. Nature 408, 936-943 (2000).

46. Nakagawa, T., Cheng, Y., Ramm, E., Sheng, M. \& Walz, T. Structure and different conformational states of native AMPA receptor complexes. Nature 433, 545-549 (2005).

47. Nicoll, R. A., Tomita, S. \& Bredt, D. S. Auxiliary subunits assist AMPA-type glutamate receptors. Science 311, 1253-1256 (2006).

48. Ziff, E. B. TARPs and the AMPA receptor trafficking paradox. Neuron 53, 627-633 (2007).

49. Schwenk, J. et al. Functional proteomics identify cornichon proteins as auxiliary subunits of AMPA receptors. Science 323, 1313-1319 (2009).

50. Herring, B. E. et al. Cornichon proteins determine the subunit composition of synaptic AMPA receptors. Neuron 77, 1083-1096 (2013).

51. Shepherd, J. D. \& Huganir, R. L. The cell biology of synaptic plasticity: AMPA receptor trafficking. Annu. Rev. Cell Dev. Biol. 23, 613-643 (2007).

52. Clem, R. L. \& Barth, A. Pathway-specific trafficking of native AMPARs by in vivo experience. Neuron 49, 663-670 (2006).

53. Plant, K. et al. Transient incorporation of native GluR2-lacking AMPA receptors during hippocampal long-term potentiation. Nat. Neurosci. 9, 602-604 (2006).

54. Liu, S. J. \& Zukin, R. S. Ca2 + -permeable AMPA receptors in synaptic plasticity and neuronal death. Trends Neurosci. 30, 126-134 (2007).

55. Clem, R. L. \& Huganir, R. L. Calcium-permeable AMPA receptor dynamics mediate fear memory erasure. Science 330, 1108-1112 (2010).

56. Shepherd, J. D. Memory, plasticity and sleep-A role for calcium permeable AMPA receptors? Front. Mol. Neurosci. 5, 49 (2012).

57. Kwak, S. \& Weiss, J. H. Calcium-permeable AMPA channels in neurodegenerative disease and ischemia. Curr. Opin. Neurobiol. 16, 281-287 (2006).

58. Aoto, J., Nam, C. I., Poon, M. M., Ting, P. \& Chen, L. Synaptic signaling by all-trans retinoic acid in homeostatic synaptic plasticity. Neuron 60, 308-320 (2008).

59. Weide, T. et al. The Golgi matrix protein GM130: a specific interacting partner of the small GTPase rablb. EMBO Rep. 2, 336-341 (2001).

60. Nakamura, N. Emerging new roles of GM130, a cis-Golgi matrix protein, in higher order cell functions. J. Pharmacol. Sci. 112, 255-264 (2010).

61. Presley, J. F. et al. ER-to-Golgi transport visualized in living cells. Nature 389, 81-85 (1997).

62. Kapitein, L. C. et al. Mixed microtubules steer dynein-driven cargo transport into dendrites. Curr. Biol. 20, 290-299 (2010).

63. Lindsay, A. J. et al. Identification and characterization of multiple novel Rab-myosin Va interactions. Mol. Biol. Cell 24, 3420-3434 (2013).

64. Schlager, M. A. \& Hoogenraad, C. C. Basic mechanisms for recognition and transport of synaptic cargos. Mol. Brain 2, 25 (2009).

65. Christoforidis, S., McBride, H. M., Burgoyne, R. D. \& Zerial, M. The Rab5 effector EEA1 is a core component of endosome docking. Nature 397, 621-625 (1999).
66. Benjamini, Y. Discovering the false discovery rate. J. R. Stat. Soc. 72, 405-416 (2010).

67. Sali, A. \& Blundell, T. L. Comparative protein modelling by satisfaction of spatial restraints. J. Mol. Biol. 234, 779-815 (1993).

68. Mintseris, J. et al. Integrating statistical pair potentials into protein complex prediction. Proteins 69, 511-520 (2007).

69. Baguet, J., Claudon-Eyl, V. \& Gachon, A. M. Tear protein G originates from denatured tear specific prealbumin as revealed by two-dimensional electrophoresis. Curr. Eye Res. 11, 1057-1065 (1992).

\section{Acknowledgements}

We thank R. Cassinari for graphic support; ALEMBIC for the microscopy technical support; L. Naldini and L. Sergi Sergi for the help in lentiviral production; M. Zerial for his mentorship and the GST-RAB5 and pGEM-p110 $\beta$ plasmids M. Fukuda for the GST-RAB39A plasmid. H.J. Risselada for providing the coarse grained structural model of a DOPC liposome; A. Ghosh for helpful discussion. This work was supported by Comitato Telethon Fondazione ONLUS, Italy (TCP04015), Jerome Lejeune Foundation, France (776) and F. Hoffmann La Roche post-doc program, Switzerland (RPF 138).

\section{Author contributions}

M.L.M., M.G. and P.D. designed the experiments. M.G. performed cell biology experiments and with L.M. the electrophysiological experiments under the conceptual advise of M.P. and J.A.E. M.L.M. performed two-hybrid screening, biochemical, cell biology experiments and A.G. helped M.L.M. F.F. supervised RAB39B modelling and docking simulations, modelled PICK1, built the RAB39B:2PICK1:AMPAR complex. F.R. modelled RAB39B and did docking simulations and analyses. S.B., H.F., M.A. and R.H. gave conceptual advices to M.L.M. for biochemical experiments. S.G. gave conceptual advice, supervised the RPF fellowship from the industrial point of view and contributed to the manuscript. F.F., E.V.A., J.A.E., R.H. and P.D. wrote the manuscript. P.D. supervised the project.

\section{Additional information}

Supplementary Information accompanies this paper at http://www.nature.com/ naturecommunications

Competing financial interests: S.G. is a employee of F. Hoffmann La Roche and M.L.M and M.G. are recipients of a post-doc fellowship (RPF ID: 138) from F. Hoffmann La Roche.

Reprints and permission information is available online at http://npg.nature.com/ reprintsandpermissions/

How to cite this article: Mignogna, M. L. et al. The intellectual disability protein RAB39B regulates selectively GluA2 trafficking determining synaptic AMPAR composition. Nat. Commun. 6:6504 doi: 10.1038/ncomms7504 (2015).

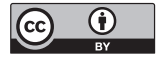

This work is licensed under a Creative Commons Attribution 4.0 International License. The images or other third party material in this article are included in the article's Creative Commons license, unless indicated otherwise in the credit line; if the material is not included under the Creative Commons license, users will need to obtain permission from the license holder to reproduce the material. To view a copy of this license, visit http://creativecommons.org/licenses/by/4.0/ 